\title{
A Multi-Scale Bioinformatics Study on Novel Mechanism of Xintong Granule for Treating Coronary Heart Disease by Network Pharmacology and Molecular Docking Verification
}

\section{Zhihong Huang}

Beijing University of Chinese Medicine

\section{Siyu Guo}

Beijing University of Chinese Medicine

Changgeng Fu

China Academy of Chinese Medical Sciences Xiyuan Hospital

\section{Wei Zhou}

Beijing University of Chinese Medicine

Jingyuan Zhang

Beijing University of Chinese Medicine

Xinkui Liu

Beijing University of Chinese Medicine

Shanshan Jia

Beijing University of Chinese Medicine

\section{Chao Wu}

Beijing University of Chinese Medicine

Shan Lu

Beijing University of Chinese Medicine

\section{Bingbing Li}

State Key Laboratory of Generic Manufacture Technology of Chinese Traditional Medicine, Linyi

\section{Zhishan Wu}

Beijing University of Chinese Medicine

\section{Yingying Tan}

Beijing University of Chinese Medicine

\section{Xiaotian Fan}

Beijing University of Chinese Medicine

\section{Guoliang Cheng}

State Key Laboratory of Generic Manufacture Technology of Chinese Traditional Medicine, Linyi Jiarui Wu ( $\nabla$ exogamy@163.com ) 
Beijing University of Traditional Chinese Medicine: Beijing University of Chinese Medicine https://orcid.org/0000-0002-1617-6110

\section{Research}

Keywords: Xintong Granule, Network pharmacology, Coronary heart disease, Signaling pathway, Molecular docking verification

Posted Date: December 1st, 2020

DOl: https://doi.org/10.21203/rs.3.rs-116130/v1

License: (9) This work is licensed under a Creative Commons Attribution 4.0 International License. Read Full License 


\section{Abstract}

Background: Xintong Granule (XTG) is a Chinese patent medicine composed of 13 Chinese herbs, which is widely used in the treatment of coronary heart disease (CHD). However, there are few studies on it, and its potential pharmacological mechanism needs to be further elucidated.

Methods: In this study, network pharmacology was employed to construct the drug-compounds-targetspathways molecular regulatory network of the treatment of $\mathrm{CHD}$ to explore the effective compounds of XTG and its underlying pharmacological mechanism. First, we established the related ingredients and potential targets of these ingredients databases by Traditional Chinese Medicine Systems Pharmacology Database (TCMSP) and A Bioinformatics Analysis Tool for Molecular mechANism of Traditional Chinese Medicine (BATMAN-TCM). Next, the CHD targets were obtained in DigSee, OMIM, DisGeNET, TTD, GeneCards and GenCLiP3 database. Then, protein-protein interaction (PPI) analysis, GO and KEGG pathway enrichment analysis were carried out and the core targets were filtered by topology. Moreover, molecular docking was performed to assess the binding potential of hub targets and key compounds.

Results: The result reflected that 178 components of XTG and 669 putative therapeutic targets were screened out. After a systematic and comprehensive analysis, we identified 9 hub targets (TNF, MAPK1, STAT3, IL6, AKT1, INS, EGFR, EGF, TP53) primarily participated in the comprehensive therapeutic effect related to blood circulation, vascular regulation, cell membrane region, compound binding, receptor activity, signal transduction, AGE-RAGE signaling pathway in diabetic complications, JAK-STAT signaling pathway, PI3K-AKT signaling pathway and MAPK signaling pathway.

Conclusion: The results of this study tentatively clarified the potential targets and signaling pathways of XTG against CHD, which may benefit to the development of clinical experimental research and application.

\section{Background}

American Heart Association statistics declared that cardiovascular and cerebrovascular diseases are the main reason of death, which bring great sufferings to the people of the world[1-3]. Narrowing of small blood vessels that supply oxygen and blood to heart may contribute to coronary heart disease (CHD), $\mathrm{CHD}$ is also called coronary artery disease (CAD). Its basic pathological manifestations are vascular and myocardial dysfunction caused by ischemia, hypoxia, oxidative stress, inflammation and apoptosis. CHD is clinically caused by the accumulation of heart artery plaques which were formed of fatty material and other substances, and it may also be called hardening of the arteries [4]. Previous researches have shown that the incidence rate of $\mathrm{CHD}$ is different from men to women, and the prognosis of female is worse than that of male [5,6]. In addition, there are many factors associated with CHD, such as psychological stress, work-related stress, lifestyle and so on [7-10]. CHD is usually prevented and treated by normalization of blood lipid profile, hypertension control, nutritional supplements in clinic [11-14]. Some studies illustrated 
that $\mathrm{CHD}$ can also be prevented by weight management, moderate alcohol intake coupled with exercise [15-17].

Traditional Chinese Medicine (TCM) has a long history of use as a traditional remedy for many diseases. It has been proved by clinical practice and has made great contributions to modern medicine [18-20]. XTG has been widely used in the treatment of CHD in China, which is made from 13 Chinese herbs, such as Salvia miltiorrhiza Bunge. (DS; DAN SHEN; family: Labiatae), Epimedium brevicornu Maxim. (YYH; YIN YANG HUO; family: Ranunculaceae) and Ophiopogon japonicus (Linn. f.) Ker-Gawl. (MD; MAI DONG; family: Liliaceae).

Network pharmacology is a new subject based on the theory of system biology, which analyzes the network of biological system and selects specific signal nodes to carry out multi-target drug molecular design. It emphasizes the multi-channel regulation of signaling pathways, improving the therapeutic effect of drugs and reducing the side effects, so as to improve the success rate of clinical trials of new drugs and save the research and development costs of drugs. With the rise of network pharmacology, its holistic and systematic characteristics are consistent with the holistic view of TCM and the principle of dialectical treatment, which has been widely used in the research of TCM [21-24]. In the present study, we identified the underlying targets and pathways of XTG in the treatment of CHD applying the network pharmacology approach, and systematically clarified the mechanism of XTG against CHD. The detailed workflow was shown in Fig. 1.

\section{Results}

\section{Compound-putative target network}

There were 178 compounds and 669 compound-associated targets collected as candidate compounds and targets from XTG by screening and deleting duplicate data (Supplementary Table 1). As the main component of XTG, DS can improve blood circulation, which contains 59 compounds. Through the analysis of XTG compound-predicted target network, it was found that the number of nodes was 847 (178 compound nodes, 669 compound-associated target nodes), and the number of edges was 2769 . Due to the excessive number of nodes and edges, only the compounds with the top 10 degrees and their related targets were shown (Figure 2(a)). As shown in Figure 2(a), a single target can be affected by multiple compounds to produce biological effects, which may play an important part in the treatment of CHD. For instance, IL6 was regulated by quercetin, luteolin and fisetin.

\section{XTG-CHD PPI network}

A total of 8860 CHD-related target were obtained from OMIM, TTD, DigSee, DisGeNET, GeneCards and GenCLiP3 database. By intersecting the XTG compound-predicted targets and the CHD targets, 493 XTG/CHD putative therapeutic targets were acquired (Supplementary Table 2 and Figure 2(b)). Only the compounds with the top 10 degrees and their associated XTG/CHD putative therapeutic targets were shown (Figure 2(c)). Whereafter, these targets were uploaded to the STRING 11.0 database to construct 
the PPI network (Supplementary Table 3 and Figure 3(a)). The network consisted of 464 nodes and 3955 edges, the degree was increasing from purple to yellow and thicker edges was defined as the stronger interactions. A total of 35 targets were obtained on the basis of average degree value $>23$, average betweenness $>0.0045$ and average closeness $>0.36$. In the present study, we consulted the literature and screened these targets. Finally, 9 targets with high degree were selected as the underlying hub targets for further analyze (Table 1). The results of topological analysis showed that the top mutual target proteins had a variety of beneficial biological function in the treatment of $\mathrm{CHD}$ at the molecular level.

\section{Module analysis and functional enrichment analysis}

Network module or cluster is a group of highly interrelated nodes, which helps to uncover the potential biological information in the network [25]. The XTG-CHD PPI network was classified to 24 clusters so as to identify the underlying mechanism of the 9 hub targets (Supplementary Table 4). Finally, 3 modules with a score of $\geq 10.0$ were picked out (Figure $4(\mathbb{(})$ )). And there were 9 hub targets (TNF, MAPK1, TP53, EGFR, IL6, EGF, INS, STAT3, AKT1) gathered in Module 2.

To deepen understand the molecular function (MF), cellular component (CC) and biological processes (BP) that are affected in CHD, we carried out $\mathrm{GO}$ enrichment analysis ( $p$-value $<0.01$ and $q$-value $<0.05$ ) for the identified XTG-CHD PPI network and 3 hub modules (Figure 3(b) and Figure 4( () ). The results demonstrated that module 1 was highly related to signal transduction, secretory regulation, cell membrane region, neuronal structure, compound binding and receptor activity. Module 2 was strongly bound up with blood circulation, vascular regulation, cell membrane region, signal transduction, compound binding and receptor activity. Module 3 was closely correlated with vascular regulation, signal transduction, compound binding and receptor activity (Supplementary Table 5 and Supplementary Table 6). Above all, the underlying targets were highly associated with blood circulation, vascular regulation, cell membrane region, signal transduction, compound binding, receptor activity.

Next, we performed KEGG pathway enrichment analysis of the XTG-CHD PPI network and 3 modules ( $p$ value $<0.05$ and $q$-value $<0.05$ ) (Figure 3(c) and Figure $4(\mathbb{(})$ ). The results indicated that module 1 was highly bound up with signal transduction and cellular processes. Module 2 was strongly related to inflammation, cancer, signal transduction, cardiovascular diseases, human diseases and infectious diseases. Module 3 was closely correlated with signal transduction, cellular processes, secretory regulation and vascular regulation (Supplementary Table 7 and Supplementary Table 8). Overall, we identified 52 CHD-related signaling pathways, AGE-RAGE signaling pathway in diabetic complications, MAPK signaling pathways, JAK-STAT signaling pathways, PI3K-AKT signaling pathways and so on. Accordingly, it is suggested that XTG may be involved in above-mentioned BP, CC and MF and signaling pathway to treat CHD.

\section{Drug-key compounds-hub targets-pathway network construction}

We utilized Cytoscape 3.8.0 software to construct a drug-key compounds-hub targets-pathway network in order to systematically and all-sidedly interpret the mechanism of XTG in treating CHD (Figure 5). As 
shown in Figure 5, there were 52 nodes and 178 edges. Those pathways were closely interacted with 9 hub targets (TNF, MAPK1, TP53, EGFR, IL6, EGF, INS, STAT3, AKT1). Luteolin and nobiletin were the compounds with the highest degree value (degree $=7$ ). AKT1 was the target with the highest degree value (degree = 33). MAPK signaling pathway and PI3K-AKT signaling pathway were the KEGG pathway with the highest degree value (hsa04010, hsa04151, degree $=7$ ). Nevertheless, the AGE-RAGE signaling pathway in diabetic complications (hsa04933) with the smallest $p$-value and $q$-value will be deeply analyzed as a significant pathway (Figure 6).

\section{Molecular docking verification}

In the present study, molecular docking was performed to verify the docking of 5 potential targets with 10 corresponding compounds in the AGE-RAGE signaling pathway in diabetic complications, and the docking results were analyzed. These 10 ingredients were observed to get into the active pocket of protein (Table 2 and Figure 7). Taking the top two predicted target-compound pairs by affinity $(\mathrm{kcal} / \mathrm{mol})$ were analyzed as an example (Table 3). Luteolin small molecule primarily forms 6 hydrogen bonds with GLN102, GLU-104, SER-99 and GLU-116 residues on TNF. Kaempferol small molecule mainly forms 7 hydrogen bonds with PRO-100, GLU-116, GLN-102, SER-100 and TRY-115 residues on TNF.

\section{Discussion}

TCM is a multi-component and multi-target complex system, which has been used to prevent and treat various cardiovascular diseases for a long time [26-28]. XTG has a good curative effect on CHD, but its pharmacological mechanism is still unknown. Therefore, in this study, we adopted network pharmacology method to identify bioactive ingredients, underlying targets and regulatory pathways of XTG against CHD.

By analyzing the module and network topology, we found 9 potential hub targets: TNF, MAPK1, TP53, EGFR, IL6, EGF, INS, STAT3, AKT1. Atherosclerosis, a progressive and chronic inflammatory process of arterial wall thickening, is the main cause of CHD. With the development of the disease, the interaction between immune cells and residual vascular wall cells ultimately result in the formation of atherosclerotic plaque. Then the lumen of the coronary artery becomes narrower and the patient will have intermittent or persistent angina pectoris. Plaque rupture leads to the formation of thrombus, which causes myocardial infarction and even death due to blood flow stop [29]. Zhang et al. and Li et al. had shown that the proinflammatory cytokines in CHD patients were significantly higher than healthy people [30, 31]. Liu et al. and Dong et al. showed that apoptosis and autophagy are involved in the pathogenesis, development and prognosis of $\mathrm{CHD}$, both enhanced in patients with $\mathrm{CHD}[32,33]$. TNF-a increases the expression of tissue factor in monocytes/macrophages to enhance thrombotic activity. At the same time, TNF-a can lead to plaque instability by increasing the expression of metalloproteinase gene and the activation of metalloproteinase through the regulation of plasmin [34]. The increase of IL-6 level in serum can upregulate the expression of fibrinogen, promote coagulation state and enhance plaque formation [35]. Abnormal proliferation and migration of vascular smooth muscle cells (VSMCs) can promote the 
pathogenesis of atherosclerosis. Mir-155-5p inhibits proliferation, migration and invasion of VSMCs and human umbilical vein endothelial cells (HUVECs) by regulating AKT1 [36]. MAPK1 mediates cell autophagy by influencing mTOR signaling, and then affect CHD process. The result of qRT-PCR illustrated that the expression of MAPK1 in CHD blood samples and endothelial progenitor cells (EPCs) were markedly up-regulated [37]. TP53 can regulate the growth of VSMCs. The growth of VSMCs was caused by the loss of TP53 activity, while the increase of TP53 level can lead to apoptosis of VSMCs. Plaque VSMCs are more susceptible to apoptosis mediated by TP53 than normal VSMCs [38]. The research hypothesized that the serum concentrations of EGF and other cytokines/growth factors may play a significant role in the pathogenesis of CHD, which the final conclusion was as follow: the concentration of EGF in serum of patients with CHD was higher than that in normal people [39]. The inflammatory mediating process and oxidative stress level were affected by inhibiting the expression of EGFR and activation of MAPK signal, thus result in the reduction of oxidative stress and anti-inflammatory [40]. The level of some cytokines in the serum of patients with CHD increased, which activated JAK-STAT signaling pathway and significantly increased the expression of STAT3. As a transcription factor, STAT3 can upregulate inflammatory factors when the expression is increased, which further explains the reason for the increased level of inflammatory markers in patients with CHD [41]. Insulin resistance is the main factor for the abnormal development of atherosclerosis and diabetes mellitus complicated with CHD. The expression level of INS can affect the condition of diabetic patients with CHD [42]. According to the results of the present study and other studies, we preliminarily hypothesized that XTG can treat CHD by regulating TNF, IL6, STAT3, AKT1 and MAPK1.

In this study, we performed GO and KEGG functional enrichment analysis to comprehend the underlying biological mechanism of XTG against CHD. A total of $52 \mathrm{CHD}$-related signaling pathways were identified through the KEGG pathway analysis ( $p$-value $<0.05$, $q$-value $<0.05$ ), such as AGE - RAGE signaling pathway in diabetic complications, MAPK signaling pathways, JAK-STAT signaling pathways and PI3KAKT signaling pathways. Consequently, these pathways may be participated in the process of CHD. AGE - RAGE signaling pathway in diabetic complications was selected as the most important signaling pathway for further analyze on the basic of the smallest $p$-value and $q$-value. MAPK1 and AKT1 are the hub genes in MAPK and PI3K-AKT signaling pathways, which can activate transcription factor NF-KB and AP-1. NF-KB may play an important role in plaque instability by regulating various stimulatory proinflammatory genes and promoting the cascade expression of procoagulant genes [43,44]. Activated AP-1 can bind to the AP-1 site in the promoter of TNF - $a$ and IL- 6 genes then regulate their expression. Subsequently, NF-KB and AP-1 transcriptionally activated TNF- $a$ and IL-6, then produce inflammation [45, 46]. Some studies have shown that the expression of TNF- $a$ and IL-6 can lead to vascular endothelial dysfunction in inflammatory coronary circulation in mice or rats, and develop into CHD $[47,48]$. STAT3, as a signal transducer and activator of transcription in JAK-STAT signaling pathway, plays an important role in vascular remodeling. The RAGE-dependent activation of STAT3 in VSMCs can activate Pim-1/NFAT axis, then NFATc1 mediate the proliferation and migration of VSMCs and promote vascular remodeling $[49,50]$. Thus, inhibition of STAT3 signal can reduce the proliferation and migration of VSMCs, then 
prevent vascular remodeling $[51,52]$. In conclusion, these results demonstrate that XTG may play a therapeutic role by regulating AGE - RAGE signaling pathway in diabetic complications.

In the present study, we adopted GO enrichment analysis to analyze the modules. These potential targets (such as TNF, AKT1, STAT3) are closely related to blood circulation, vascular regulation, cell membrane region, compound binding, receptor activity and signal transduction. These results indicate that XTG is involved in these $\mathrm{BP}, \mathrm{CC}$ and $\mathrm{MF}$ to treat $\mathrm{CHD}$.

Molecular docking verification provided an intuitive explanation for the interaction between key compounds and their underlying protein targets. For instance, luteolin small molecule mainly forms 6 hydrogen bonds with GLN-102, GLU-104, SER-99 and GLU-116 residues on TNF. Luteolin is an important flavonoid, which has strong anti-cancer, anti-inflammatory activity, and it is also used as a neuroprotective agent $[53,54]$. In vitro, in vivo and clinical studies have shown that the main pharmacological mechanism of luteolin is its anti-inflammatory activity, which is by regulating the signaling pathways and the transcription factors such as MAPK signaling pathway, JAK-STAT signaling pathway, STAT3, NF-KB and AP-1 [55]. Generally, it is presumed that the essential ingredients of XTG may play an important part in the remedy for $\mathrm{CHD}$ through hub targets in these top-ranking signaling pathways. However, there are some limitations in our study. For instance, the results are the known chemical components, related targets and signal pathways of XTG screened from literature and existing databases. Therefore, it is necessary to conduct a more in-depth study on its potential mechanism.

\section{Conclusion}

A total of 178 components and 669 known therapeutic targets of XTG were collected and explored the potential mechanism of XTG treating CHD by network pharmacology and molecular docking verification. XTG has therapeutic effect on CHD by regulating 9 hub targets: TNF, MAPK1, TP53, EGFR, IL6, EGF, INS, STAT3, AKT1. On basis of GO and KEGG pathway enrichment analysis, we discovered that these hub targets treat $\mathrm{CHD}$ by involving in blood circulation, vascular regulation, cell membrane region, compound binding, receptor activity and signal transduction. In summary, the results of the study tentatively forecasted the potential mechanism of XTG in the treatment of CHD, and proved the characteristics of multi-target synergy. However, the mechanism of XTG against CHD needs further animal experiments, molecular biology experiments and clinical studies.

\section{Methods}

\section{Identification of XTG compounds and targets}

The chemical components and potential targets of XTG were obtained from Traditional Chinese Medicine Systems Pharmacology Database (TCMSP, https://tcmspw.com/tcmsp.php) [56] and A Bioinformatics Analysis Tool for Molecular mechANism of Traditional Chinese Medicine (BATMAN-TCM, https://bionet.ncpsb.org/batman-tcm/) [57]. And then, the UniProt (https://www.uniprot.org/) [58] was 
applied to transform the protein name of the XTG active compounds into the gene names. Finally, we screened the search results and persist only the researches related to "Homo sapiens (Human)", then standardized the names and deleted the duplicate data.

\section{Collection of target proteins associated with CHD}

"CHD" and "coronary heart disease" were used as the keywords to upload to the DigSee (https://210.107.182.61/geneSearch/) [59], OMIM (https://omim.org/) [60], DisGeNET (https://www.disgenet.org/search) [61], Therapeutic Target Database (TTD, https://db.idrblab.net/ttd/) [62], GeneCards (https://www.genecards.org/) [63] and GenCLiP3 (https://ci.smu.edu.cn/genclip3/analysis.php) [64]. Moreover, the species was designated as "Homo sapiens (Human)". We retained the intersection between the CHD-related human gene and the target gene of the XTG bioactive ingredients for further analysis.

\section{Protein-protein interaction (PPI) network construction}

Submit the names of XTG/CHD putative therapeutic targets to STRING 11.0 database (https://stringdb.org) [65], which stores information about protein-protein interactions. And only "Homo sapiens (Human)" proteins with the minimum required interaction score higher than 0.7 were screened out.

\section{Network establishment and module analysis}

In order to describe the therapeutic mechanisms of XTG on CHD from the perspective of network targets, the Cytoscape 3.8.0 (https://cytoscape.org/) [66] was utilized to construct five visualization networks as follows: (1) XTG compound-predicted target network; (2) Compound-XTG/CHD putative therapeutic target network; (3) XTG-CHD PPI network; (4) Module analysis network; (5) Drug-key compounds-hub targetspathways network. The "degree" is considered as the number of edges connected to the node. The "edges" represents the interaction, association, or any other well-defined relationship. In addition, the "betweenness" is defined as the number of shortest paths through a given node. Moreover, the "closeness" characterizes the reciprocal of the sum of the distances from one node to another [67-71]. The higher the quantitative value of a node's parameters in network (such as degree, betweenness, and closeness), the more significant the node is.

Apply Molecular Complex Detection (MCODE) [72] algorithms to seek out the interaction intensive areas in PPI network according to complex connection data. In this study, we recognized the high-density regions of XTG-CHD PPI network on the basis of the predetermined parameters of MCODE (Degree Cutoff=2; Node Score Cutoff=0.2; K-Core=2; Max. Depth=100) [73] and analyzed the core genes in each important module.

\section{Functional enrichment analysis}

The R 3.6.1 software with the Bioconductor package was operated to assess the effect of underlying hub targets by bioinformatic annotation, which consist of Gene Ontology (G0) knowledgebase 
(http://geneontology.org/), Kyoto Encyclopedia of Genes and Genomes (KEGG) pathway enrichment analysis (https://www.genome.jp/kegg/) [74-76].

\section{Molecular docking verification}

First, we set the number of rotatable bonds of 10 key small molecular compounds related to 5 hub targets in AGE-RAGE signaling pathway in diabetic complications by AutoDockTools 1.5.6 [77]. Then, the hub target protein structures were obtained from the Protein Data Bank database (PDB, https://www.rcsb.org/) [78]. The screening conditions were as follows: (1) the protein structure is obtained by X-ray diffraction; (2) the crystal resolution of protein is less than $3 \AA$; (3) the protein structure reported in literature of molecular docking is preferred; (4) the organism is Homo sapiens. A total of 5 hub target protein PDB IDs were collected on account of the above conditions. In the meantime, utilizing

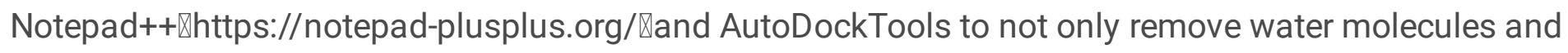
pro-ligand small molecules, but also hydrogenate and charge. Ultimately, AutoDock Vina 1.1.2 was applied to carry on molecular docking verification [79], and the PyMOL 2.4 .0 (https://pymol.org/2/) was employed to analyze the visual docking results $[80,81]$.

\section{Abbreviations}

TCM: Tradition Chinese Medicine

CHD: coronary heart disease

CAD: coronary artery disease

XTG: Xintong Granule

TTD: Therapeutic Target Database

OMIM: Online Mendelian Inheritance in Man

DigSee: Disease Gene Search Engine with Evidence Sentences

AKT1: RAC-alpha serine/threonine-protein kinase

TP53: Cellular tumor antigen p53

INS: Insulin

MAPK1: Mitogen-activated protein kinase 1

IL6: Interleukin-6

EGFR: Epidermal growth factor receptor 
EGF: Pro-epidermal growth factor

TNF: Tumor necrosis factor

STAT3: Signal transducer and activator of transcription 3

NF-kB: Nuclear factor kappa-B

AP-1: Activator protein 1

Pim-1: Serine/threonine-protein kinase pim-1

NFATc1: Nuclear factor of activated T-cells

\section{Declarations}

\section{Availability of data and materials statement}

The raw data supporting the conclusions of this article will be made available by the authors, without undue reservation. All data obtained or analyzed during this study are available from the published article and its supplementary information files. The datasets during the current study are available from the corresponding author upon reasonable request.

\section{Funding}

This work was supported by the Young Scientists Training Program of Beijing University of Chinese Medicine and the National Nature Science Foundation of China (Grant nos. 81673829).

\section{Acknowledgements}

This work was supported by the Young Scientists Training Program of Beijing University of Chinese Medicine and the National Nature Science Foundation of China (Grant nos. 81673829).

\section{Authors' contributions}

ZH and JW conceived and designed the study. SG, CF, WZ, XL and JZ provided significative suggestions on the methodology. SL, ZW, YT and XF collected the data. CW, SJ, GC and BL performed the data analysis, $\mathrm{ZH}$ and JW wrote and revised the manuscript. All authors were responsible for reviewing the data. All authors read and approved the final manuscript.

\section{Ethics declarations}

\section{Ethics approval and consent to participate}

Not applicable. 


\section{Consent for publication}

Not applicable.

\section{Competing interests}

The authors declare no competing interests in any respect.

\section{References}

1. Dalen JE, Alpert JS, Goldberg RJ, Weinstein RS. The epidemic of the 20(th) century: coronary heart disease. Am J Med. 2014;127(9):807-812. doi:10.1016/j.amjmed.2014.04.015.

2. Virani SS, Alonso A, Benjamin EJ, Bittencourt MS, Callaway CW, Carson AP, et al. Heart Disease and Stroke Statistics-2020 Update: A Report From the American Heart Association. Circulation. 2020;141(9):e139-e596. doi:10.1161/CIR.0000000000000757.

3. Xu S, Xu Y, Liu P, Zhang S, Liu H, Slavin S, et al. The novel coronary artery disease risk gene JCAD/KIAA1462 promotes endothelial dysfunction and atherosclerosis. Eur Heart J. 2019;40(29):2398-2408. doi:10.1093/eurheartj/ehz303.

4. Emanuele DA, Alexander T, Frances W, John D. Coronary Heart Disease. IARC Sci Publ. 2011; (163):363-386.

5. Mehta PK, Wenger NK. Coronary Heart Disease in Women: Battle is Won, but the War Remains. Minerva Med. 2007;98(5):459-478.

6. Khamis RY, Ammari T, Mikhail GW. Gender differences in coronary heart disease. Heart. 2016;102(14):1142-1149. doi:10.1136/heartjnl-2014-306463.

7. Sara JD, Prasad M, Eleid MF, Zhang M, Widmer RJ, Lerman A. Association Between Work-Related Stress and Coronary Heart Disease: A Review of Prospective Studies Through the Job Strain, EffortReward Balance, and Organizational Justice Models. J Am Heart Assoc. 2018;7(9). doi:10.1161/JAHA.117.008073.

8. Ulbricht TLV, Southgate DAT. Coronary heart disease: seven dietary factors. The Lancet. 1991;338(8773):985-992. doi:10.1016/0140-6736(91)91846-m.

9. Stewart RAH, Held C, Hadziosmanovic N, Armstrong PW, Cannon CP, Granger CB, et al. Physical Activity and Mortality in Patients With Stable Coronary Heart Disease. J Am Coll Cardiol. 2017;70(14):1689-1700. doi:10.1016/j.jacc.2017.08.017.

10. Kubareva MI, Ibatov AD. [Psychosocial factors and coronary heart disease]. Ter Arkh. 2019;91(12):7074. doi:10.26442/00403660.2019.12.000086.

11. Escobar E. Hypertension and coronary heart disease. J Hum Hypertens. 2002;16 Suppl 1:S61-63. doi:10.1038/sj.jhh.1001345.

12. Houston M, Minich D, Sinatra ST, Kahn JK, Guarneri M. Recent Science and Clinical Application of Nutrition to Coronary Heart Disease. J Am Coll Nutr. 2018;37(3):169-187. 
doi:10.1080/07315724.2017.1381053.

13. Houston M. The role of noninvasive cardiovascular testing, applied clinical nutrition and nutritional supplements in the prevention and treatment of coronary heart disease. Ther Adv Cardiovasc Dis. 2018;12(3):85-108. doi:10.1177/1753944717743920.

14. Kindwall KE. Therapy for Coronary Heart Disease in Women. Cardiovascular clinics. 1989;19(3):195203.

15. Mukamal KJ, Rimm EB. Alcohol's Effects on the Risk for Coronary Heart Disease. Alcohol Res Health. 2001;25(4):255-261.

16. Yusuf S, Hawken S, Ôunpuu S, Dans T, Avezum A, Lanas F, et al. Effect of potentially modifiable risk factors associated with myocardial infarction in 52 countries (the INTERHEART study): case-control study. The Lancet. 2004;364(9438):937-952. doi:10.1016/s0140-6736(04)17018-9.

17. Liu T, Chan AW, Liu YH, Taylor-Piliae RE. Effects of Tai Chi-based cardiac rehabilitation on aerobic endurance, psychosocial well-being, and cardiovascular risk reduction among patients with coronary heart disease: A systematic review and meta-analysis. Eur J Cardiovasc Nurs. 2018;17(4):368-383. doi:10.1177/1474515117749592.

18. Oravecz M, Meszaros J. [Traditional Chinese medicine: theoretical background and its use in China]. Orv Hetil. 2012;153(19):723-731. doi:10.1556/OH.2012.29365.

19. Wang J, Wong YK, Liao F. What has traditional Chinese medicine delivered for modern medicine? Expert Rev Mol Med. 2018;20:e4. doi:10.1017/erm.2018.3.

20. Cyranoski D. Why Chinese medicine is heading for clinics around the world. Nature. 2018;561(7724):448-450. doi:10.1038/d41586-018-06782-7.

21. Yuan H, Ma Q, Cui H, Liu G, Zhao X, Li W, et al. How Can Synergism of Traditional Medicines Benefit from Network Pharmacology? Molecules. 2017;22(7). doi:10.3390/molecules22071135.

22. Song Y, Wang H, Pan Y, Liu T. Investigating the Multi-Target Pharmacological Mechanism of Hedyotis diffusa Willd Acting on Prostate Cancer: A Network Pharmacology Approach. Biomolecules. 2019;9(10). doi:10.3390/biom9100591.

23. Boezio B, Audouze K, Ducrot P, Taboureau O. Network-based Approaches in Pharmacology. Mol Inform. 2017;36(10). doi:10.1002/minf.201700048.

24. Li S, Zhang B. Traditional Chinese medicine network pharmacology: theory, methodology and application. Chinese Journal of Natural Medicines. 2013;11(2):110-120. doi:10.1016/s18755364(13)60037-0.

25. Zuo H, Zhang Q, Su S, Chen Q, Yang F, Hu Y. A network pharmacology-based approach to analyse potential targets of traditional herbal formulas: An example of Yu Ping Feng decoction. Sci Rep. 2018;8(1):11418. doi:10.1038/s41598-018-29764-1.

26. Hao DC, Xiao PG. Impact of Drug Metabolism/Pharmacokinetics and their Relevance Upon Traditional Medicine-based Cardiovascular Drug Research. Curr Drug Metab. 2019;20(7):556-574. doi:10.2174/1389200220666190618101526. 
27. Shu Z, Wu T, Shahen M, Guo Z, Shu J, Wang HE, et al. System-Pharmacology Dissection of Traditional Chinese herbs SINI Decoction for Treatment of Cardiovascular Diseases. An Acad Bras Cienc. 2019;91(3):e20180424. doi:10.1590/0001-3765201920180424.

28. Hao P, Jiang F, Cheng J, Ma L, Zhang Y, Zhao Y. Traditional Chinese Medicine for Cardiovascular Disease: Evidence and Potential Mechanisms. J Am Coll Cardiol. 2017;69(24):2952-2966. doi:10.1016/j.jacc.2017.04.041.

29. Wirtz PH, von Kanel R. Psychological Stress, Inflammation, and Coronary Heart Disease. Curr Cardiol Rep. 2017;19(11):111. doi:10.1007/s11886-017-0919-x.

30. Li X, Zhang F, Zhou H, Hu Y, Guo D, Fang X, et al. Interplay of TNF-alpha, soluble TNF receptors and oxidative stress in coronary chronic total occlusion of the oldest patients with coronary heart disease. Cytokine. 2020;125:154836. doi:10.1016/j.cyto.2019.154836.

31. Zhang J, Jin J, Liu J, He Y, Zhang P, Ye W, et al. A study of the correlation of insulin resistance and leptin with inflammatory factors and vascular endothelial injury in T2DM patients with CHD. Exp Ther Med. 2018;16(1):265-269. doi:10.3892/etm.2018.6170.

32. Liu L, Cheng Z, Yang J. MiR-23 regulates cell proliferation and apoptosis of vascular smooth muscle cells in coronary heart disease. Pathol Res Pract. 2018;214(11):1873-1878. doi:10.1016/j.prp.2018.09.004.

33. Dong Y, Chen H, Gao J, Liu Y, Li J, Wang J. Molecular machinery and interplay of apoptosis and autophagy in coronary heart disease. J Mol Cell Cardiol. 2019;136:27-41. doi:10.1016/j.yjmcc.2019.09.001.

34. Sullivan GW, Sarembock IJ, Linden J. The role of inflammation in vascular diseases. J Leukoc Biol. 2000;67(5):591-602. doi:10.1002/jlb.67.5.591.

35. Li H, Sun K, Zhao R, Hu J, Hao Z, Wang F, et al. Inflammatory biomarkers of coronary heart disease. Front Biosci (Schol Ed). 2018;10:185-196. doi:10.2741/s508.

36. Chen L, Zheng SY, Yang CQ, Ma BM, Jiang D. MiR-155-5p inhibits the proliferation and migration of VSMCs and HUVECs in atherosclerosis by targeting AKT1. Eur Rev Med Pharmacol Sci. 2019;23(5):2223-2233. doi:10.26355/eurrev_201903_17270.

37. Zhu Y, Yang T, Duan J, Mu N, Zhang T. MALAT1/miR-15b-5p/MAPK1 mediates endothelial progenitor cells autophagy and affects coronary atherosclerotic heart disease via mTOR signaling pathway. Aging (Albany NY). 2019;11(4):1089-1109. doi:10.18632/aging.101766.

38. Beiras-Fernandez A, Angele MK, Koutang C, Lohse P, Reichart B, Lohse P, et al. Genetic polymorphisms of TP53 and FAS promoter modulate the progression of coronary artery disease after coronary artery bypass grafting: a gender-specific view. Inflamm Res. 2011;60(5):439-445. doi:10.1007/s00011-010-0282-5.

39. Mirhafez SR, Zarifian A, Ebrahimi M, Ali RF, Avan A, Tajfard M, et al. Relationship between serum cytokine and growth factor concentrations and coronary artery disease. Clin Biochem. 2015;48(9):575-580. doi:10.1016/j.clinbiochem.2015.02.002. 
40. Li Z, Yang L, Liu Y, Xu H, Wang S, Liu Y, et al. Anti-inflammatory and antioxidative effects of Dan-Lou tablets in the treatment of coronary heart disease revealed by metabolomics integrated with molecular mechanism studies. J Ethnopharmacol. 2019;240:111911. doi:10.1016/j.jep.2019.111911.

41. Alikhah A, Pahlevan Kakhki M, Ahmadi A, Dehghanzad R, Boroumand MA, Behmanesh M. The role of Inc-DC long non-coding RNA and SOCS1 in the regulation of STAT3 in coronary artery disease and type 2 diabetes mellitus. J Diabetes Complications. 2018;32(3):258-265. doi:10.1016/j.jdiacomp.2017.12.001.

42. Hashemzadeh AA, Nasoohi N, Raygan F, Aghadavod E, Akbari E, Taghizadeh M, et al. Flaxseed Oil Supplementation Improve Gene Expression Levels of PPAR-gamma, LP(a), IL-1 and TNF-alpha in Type 2 Diabetic Patients with Coronary Heart Disease. Lipids. 2017;52(11):907-915. doi:10.1007/s11745-017-4295-5.

43. Monaco C, Andreakos E, Kiriakidis S, Mauri C, Bicknell C, Foxwell B, et al. Canonical pathway of nuclear factor kappa $B$ activation selectively regulates proinflammatory and prothrombotic responses in human atherosclerosis. Proc Natl Acad Sci U S A. 2004;101(15):5634-5639. doi:10.1073/pnas.0401060101.

44. Collins T, Cybulsky MI. NF-kappaB: pivotal mediator or innocent bystander in atherogenesis? J Clin Invest. 2001;107(3):255-264. doi:10.1172/JCl10373.

45. Annamalai G, Suresh K. [6]-Shogaol attenuates inflammation, cell proliferation via modulate NFkappaB and AP-1 oncogenic signaling in 7,12-dimethylbenz[a]anthracene induced oral carcinogenesis. Biomed Pharmacother. 2018;98:484-490. doi:10.1016/j.biopha.2017.12.009.

46. Kopp E, Ghosh S. Inhibition of NF-kappa B by sodium salicylate and aspirin. Science. 1994;265(5174):956-959. doi:10.1126/science.8052854.

47. Gao X, Zhang H, Schmidt AM, Zhang C. AGE/RAGE produces endothelial dysfunction in coronary arterioles in type 2 diabetic mice. Am J Physiol Heart Circ Physiol. 2008;295(2):H491-498. doi:10.1152/ajpheart.00464.2008.

48. Suchal K, Malik S, Khan SI, Malhotra RK, Goyal SN, Bhatia J, et al. Protective effect of mangiferin on myocardial ischemia-reperfusion injury in streptozotocin-induced diabetic rats: role of AGERAGE/MAPK pathways. Sci Rep. 2017;7:42027. doi:10.1038/srep42027.

49. Singh NK, Kundumani-Sridharan V, Kumar S, Verma SK, Kotla S, Mukai H, et al. Protein kinase N1 is a novel substrate of NFATC1-mediated cyclin D1-CDK6 activity and modulates vascular smooth muscle cell division and migration leading to inward blood vessel wall remodeling. J Biol Chem. 2012;287(43):36291-36304. doi:10.1074/jbc.M112.361220.

50. Meloche J, Paulin R, Courboulin A, Lambert C, Barrier M, Bonnet P, et al. RAGE-dependent activation of the oncoprotein Pim1 plays a critical role in systemic vascular remodeling processes. Arterioscler Thromb Vasc Biol. 2011;31(9):2114-2124. doi:10.1161/ATVBAHA.111.230573.

51. Dutzmann J, Daniel JM, Bauersachs J, Hilfiker-Kleiner D, Sedding DG. Emerging translational approaches to target STAT3 signalling and its impact on vascular disease. Cardiovasc Res. 
2015;106(3):365-374. doi:10.1093/cvr/cvv103.

52. Lu QB, Wang HP, Tang ZH, Cheng H, Du Q, Wang YB, et al. Nesfatin-1 functions as a switch for phenotype transformation and proliferation of VSMCs in hypertensive vascular remodeling. Biochim Biophys Acta Mol Basis Dis. 2018;1864(6 Pt A):2154-2168. doi:10.1016/j.bbadis.2018.04.002.

53. Nabavi SF, Braidy N, Gortzi O, Sobarzo-Sanchez E, Daglia M, Skalicka-Wozniak K, et al. Luteolin as an anti-inflammatory and neuroprotective agent: A brief review. Brain Res Bull. 2015;119(Pt A):1-11. doi:10.1016/j.brainresbull.2015.09.002.

54. Imran M, Rauf A, Abu-Izneid T, Nadeem M, Shariati MA, Khan IA, et al. Luteolin, a flavonoid, as an anticancer agent: A review. Biomed Pharmacother. 2019;112:108612. doi:10.1016/j.biopha.2019.108612.

55. Aziz N, Kim MY, Cho JY. Anti-inflammatory effects of luteolin: A review of in vitro, in vivo, and in silico studies. J Ethnopharmacol. 2018;225:342-358. doi:10.1016/j.jep.2018.05.019.

56. Ru J, Li P, Wang J, Zhou W, Li B, Huang C, et al. TCMSP: a database of systems pharmacology for drug discovery from herbal medicines. J Cheminform. 2014;6:13. doi:10.1186/1758-2946-6-13.

57. Liu Z, Guo F, Wang Y, Li C, Zhang X, Li H, et al. BATMAN-TCM: a Bioinformatics Analysis Tool for Molecular mechANism of Traditional Chinese Medicine. Sci Rep. 2016;6:21146. doi:10.1038/srep21146.

58. UniProt C. UniProt: a worldwide hub of protein knowledge. Nucleic Acids Res. 2019;47(D1):D506D515. doi:10.1093/nar/gky1049.

59. Kim J, So S, Lee HJ, Park JC, Kim JJ, Lee H. DigSee: Disease gene search engine with evidence sentences (version cancer). Nucleic Acids Res. 2013;41(Web Server issue):W510-517. doi:10.1093/nar/gkt531.

60. Amberger JS, Bocchini CA, Schiettecatte F, Scott AF, Hamosh A. OMIM.org: Online Mendelian Inheritance in Man (OMIM(R)), an online catalog of human genes and genetic disorders. Nucleic Acids Res. 2015;43(Database issue):D789-798. doi:10.1093/nar/gku1205.

61. Pinero J, Bravo A, Queralt-Rosinach N, Gutierrez-Sacristan A, Deu-Pons J, Centeno E, et al. DisGeNET: a comprehensive platform integrating information on human disease-associated genes and variants. Nucleic Acids Res. 2017;45(D1):D833-D839. doi:10.1093/nar/gkw943.

62. Wang Y, Zhang S, Li F, Zhou Y, Zhang Y, Wang Z, et al. Therapeutic target database 2020: enriched resource for facilitating research and early development of targeted therapeutics. Nucleic Acids Res. 2020;48(D1):D1031-D1041. doi:10.1093/nar/gkz981.

63. Stelzer G, Rosen N, Plaschkes I, Zimmerman S, Twik M, Fishilevich S, et al. The GeneCards Suite: From Gene Data Mining to Disease Genome Sequence Analyses. Curr Protoc Bioinformatics. 2016;54:1 30 31-31 30 33. doi:10.1002/cpbi.5.

64. Wang JH, Zhao LF, Wang HF, Wen YT, Jiang KK, Mao XM, et al. GenCLiP 3: mining human genes' functions and regulatory networks from PubMed based on co-occurrences and natural language processing. Bioinformatics. 2019. doi:10.1093/bioinformatics/btz807. 
65. Szklarczyk D, Morris JH, Cook H, Kuhn M, Wyder S, Simonovic M, et al. The STRING database in 2017: quality-controlled protein-protein association networks, made broadly accessible. Nucleic Acids Res. 2017;45(D1):D362-D368. doi:10.1093/nar/gkw937.

66. Doncheva NT, Morris JH, Gorodkin J, Jensen LJ. Cytoscape StringApp: Network Analysis and Visualization of Proteomics Data. J Proteome Res. 2019;18(2):623-632. doi:10.1021/acs.jproteome.8b00702.

67. Jeong $\mathrm{H}$, Mason SP, Barabasi AL, Oltvai ZN. Lethality and centrality in protein networks. Nature. 2001;411(6833):41-42. doi:10.1038/35075138.

68. Liu X, Wu J, Zhang D, Wang K, Duan X, Zhang X. A Network Pharmacology Approach to Uncover the Multiple Mechanisms of Hedyotis diffusa Willd. on Colorectal Cancer. Evid Based Complement Alternat Med. 2018;2018:6517034. doi:10.1155/2018/6517034.

69. Liu X, Wu J, Zhang D, Wang K, Duan X, Meng Z, et al. Network Pharmacology-Based Approach to Investigate the Mechanisms of Hedyotis diffusa Willd. in the Treatment of Gastric Cancer. Evid Based Complement Alternat Med. 2018;2018:7802639. doi:10.1155/2018/7802639.

70. Yu G, Wang W, Wang X, Xu M, Zhang L, Ding L, et al. Network pharmacology-based strategy to investigate pharmacological mechanisms of Zuojinwan for treatment of gastritis. BMC Complement Altern Med. 2018;18(1):292. doi:10.1186/s12906-018-2356-9.

71. Li S. Network Systems Underlying Traditional Chinese Medicine Syndrome and Herb Formula. Current Bioinformatics. 2009;4(3):188-196. doi:10.2174/157489309789071129.

72. Bader GD, Hogue CW. An automated method for finding molecular complexes in large protein interaction networks. BMC Bioinformatics. 2003;4:2. doi:10.1186/1471-2105-4-2.

73. Feng H, Gu ZY, Li Q, Liu QH, Yang XY, Zhang JJ. Identification of significant genes with poor prognosis in ovarian cancer via bioinformatical analysis. J Ovarian Res. 2019;12(1):35. doi:10.1186/s13048-019-0508-2.

74. The Gene Ontology C. Expansion of the Gene Ontology knowledgebase and resources. Nucleic Acids Res. 2017;45(D1):D331-D338. doi:10.1093/nar/gkw1108.

75. Kanehisa M, Furumichi M, Tanabe M, Sato Y, Morishima K. KEGG: new perspectives on genomes, pathways, diseases and drugs. Nucleic Acids Res. 2017;45(D1):D353-D361. doi:10.1093/nar/gkw1092.

76. Huber W, Carey VJ, Gentleman R, Anders S, Carlson M, Carvalho BS, et al. Orchestrating highthroughput genomic analysis with Bioconductor. Nat Methods. 2015;12(2):115-121. doi:10.1038/nmeth.3252.

77. Morris GM, Huey R, Lindstrom W, Sanner MF, Belew RK, Goodsell DS, et al. AutoDock4 and AutoDockTools4: Automated docking with selective receptor flexibility. J Comput Chem. 2009;30(16):2785-2791. doi:10.1002/jcc.21256.

78. Burley SK, Berman HM, Kleywegt GJ, Markley JL, Nakamura H, Velankar S. Protein Data Bank (PDB): The Single Global Macromolecular Structure Archive. Methods Mol Biol. 2017;1607:627-641. doi:10.1007/978-1-4939-7000-1_26. 
79. Trott O, Olson AJ. AutoDock Vina: improving the speed and accuracy of docking with a new scoring function, efficient optimization, and multithreading. J Comput Chem. 2010;31(2):455-461. doi:10.1002/jcc.21334.

80. Yuan S, Chan HCS, Filipek S, Vogel H. PyMOL and Inkscape Bridge the Data and the Data Visualization. Structure. 2016;24(12):2041-2042. doi:10.1016/j.str.2016.11.012.

81. Rigsby RE, Parker $A B$. Using the PyMOL application to reinforce visual understanding of protein structure. Biochem Mol Biol Educ. 2016;44(5):433-437. doi:10.1002/bmb.20966.

\section{Tables}

Table 1

Topological information of 9 potential hub targets

\begin{tabular}{|c|c|c|c|c|c|}
\hline $\begin{array}{l}\text { UniProt } \\
\text { ID }\end{array}$ & $\begin{array}{l}\text { Targets } \\
\text { name }\end{array}$ & Protein name & $\begin{array}{l}\text { Betweenness } \\
\text { Centrality }\end{array}$ & $\begin{array}{l}\text { Closeness } \\
\text { Centrality }\end{array}$ & Degree \\
\hline P31749 & AKT1 & $\begin{array}{l}\text { RAC-alpha serine/threonine- } \\
\text { protein kinase }\end{array}$ & 0.0634468 & 0.48938429 & 113 \\
\hline P04637 & TP53 & Cellular tumor antigen p53 & 0.05966534 & 0.48121086 & 100 \\
\hline P01308 & INS & Insulin & 0.12196118 & 0.48886532 & 91 \\
\hline P28482 & MAPK1 & $\begin{array}{l}\text { Mitogen-activated protein } \\
\text { kinase } 1\end{array}$ & 0.03848379 & 0.48020833 & 90 \\
\hline P05231 & IL6 & Interleukin-6 & 0.02979831 & 0.47476828 & 88 \\
\hline P00533 & EGFR & $\begin{array}{l}\text { Epidermal growth factor } \\
\text { receptor }\end{array}$ & 0.03015294 & 0.4637827 & 79 \\
\hline P01133 & EGF & Pro-epidermal growth factor & 0.02278369 & 0.461 & 78 \\
\hline P01375 & TNF & Tumor necrosis factor & 0.01424499 & 0.45643564 & 78 \\
\hline P40763 & STAT3 & $\begin{array}{l}\text { Signal transducer and activator } \\
\text { of transcription } 3\end{array}$ & 0.01477217 & 0.4471387 & 77 \\
\hline
\end{tabular}

Table 2 The information of 10 corresponding compounds to 5 potential hub targets 


\begin{tabular}{|c|c|c|c|}
\hline No. & Compound & $\begin{array}{l}\text { Molecular } \\
\text { Formula }\end{array}$ & Structure \\
\hline 1 & Fisetin & $\mathrm{C}_{15} \mathrm{H}_{10} \mathrm{O}_{6}$ & \\
\hline 2 & Kaempferol & $\mathrm{C}_{15} \mathrm{H}_{10} \mathrm{O}_{6}$ & \\
\hline 3 & Luteolin & $\mathrm{C}_{15} \mathrm{H}_{10} \mathrm{O}_{6}$ & \\
\hline 4 & Methyl Ophiopogonanone B & $\mathrm{C}_{19} \mathrm{H}_{20} \mathrm{O}_{5}$ & \\
\hline 5 & Naringenin & $\mathrm{C}_{15} \mathrm{H}_{12} \mathrm{O}_{5}$ & \\
\hline 6 & Ophiopogonanone E & $\mathrm{C}_{19} \mathrm{H}_{20} \mathrm{O}_{7}$ & \\
\hline 7 & Quercetin & $\mathrm{C}_{15} \mathrm{H}_{10} \mathrm{O}_{7}$ & \\
\hline 8 & Nobiletin & $\mathrm{C}_{21} \mathrm{H}_{22} \mathrm{O}_{8}$ & \\
\hline 9 & Arachidonic acid & $\mathrm{C}_{20} \mathrm{H}_{32} \mathrm{O}_{2}$ & \\
\hline 10 & Cryptotanshinone & $\mathrm{C}_{19} \mathrm{H}_{20} \mathrm{O}_{3}$ & \\
\hline
\end{tabular}

Table 3 The molecular docking results analysis 


\begin{tabular}{|c|c|c|c|c|c|}
\hline NO. & Proteins & $\begin{array}{l}\text { PDB } \\
\text { ID }\end{array}$ & Protein structure & Test compounds & $\begin{array}{c}\text { Affinity } \\
(\mathrm{kcal} / \mathrm{mol})\end{array}$ \\
\hline 1 & AKT1 & 2UZR & & $\begin{array}{c}\text { Methyl Ophiopogonanone B } \\
\text { Ophiopogonanone E } \\
\text { Luteolin } \\
\text { Naringenin } \\
\text { Kaempferol } \\
\text { Fisetin } \\
\text { Quercetin }\end{array}$ & $\begin{array}{l}-7.6 \\
-7.6 \\
-6.8 \\
-6.7 \\
-6.5 \\
-6.5 \\
-6.5\end{array}$ \\
\hline 2 & IL6 & $1 \mathrm{ALU}$ & & $\begin{array}{c}\text { Fisetin } \\
\text { Luteolin } \\
\text { Nobiletin }\end{array}$ & $\begin{array}{l}-7.1 \\
-7.0 \\
-5.8\end{array}$ \\
\hline 3 & MAPK1 & 4FMQ & & $\begin{array}{l}\text { Naringenin } \\
\text { Nobiletin } \\
\text { Luteolin } \\
\text { Arachidonic acid }\end{array}$ & $\begin{array}{l}-8.7 \\
-8.7 \\
-8.6 \\
-6.8\end{array}$ \\
\hline 4 & STAT3 & 6NJS & 5 & Cryptotanshinone & -8.0 \\
\hline 5 & TNF & $1 \mathrm{TNF}$ & & $\begin{array}{c}\text { Luteolin } \\
\text { Kaempferol } \\
\text { Fisetin } \\
\text { Cryptotanshinone } \\
\text { Nobiletin }\end{array}$ & $\begin{array}{l}-9.0 \\
-8.9 \\
-8.8 \\
-8.5 \\
-8.5\end{array}$ \\
\hline
\end{tabular}

\section{Figures}




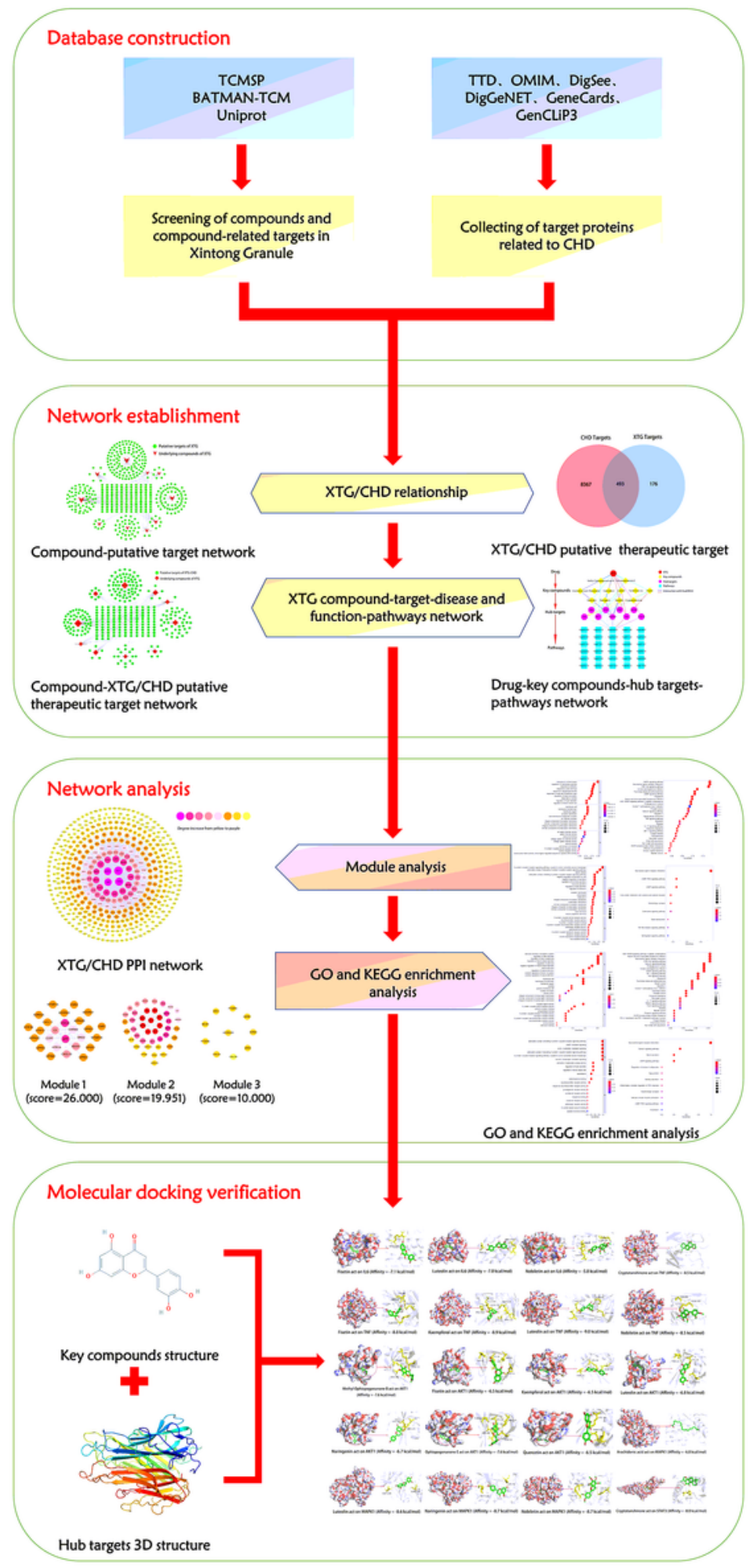

\section{Figure 1}

Network pharmacology and molecular docking verification workflow of XTG for the treatment of CHD. 


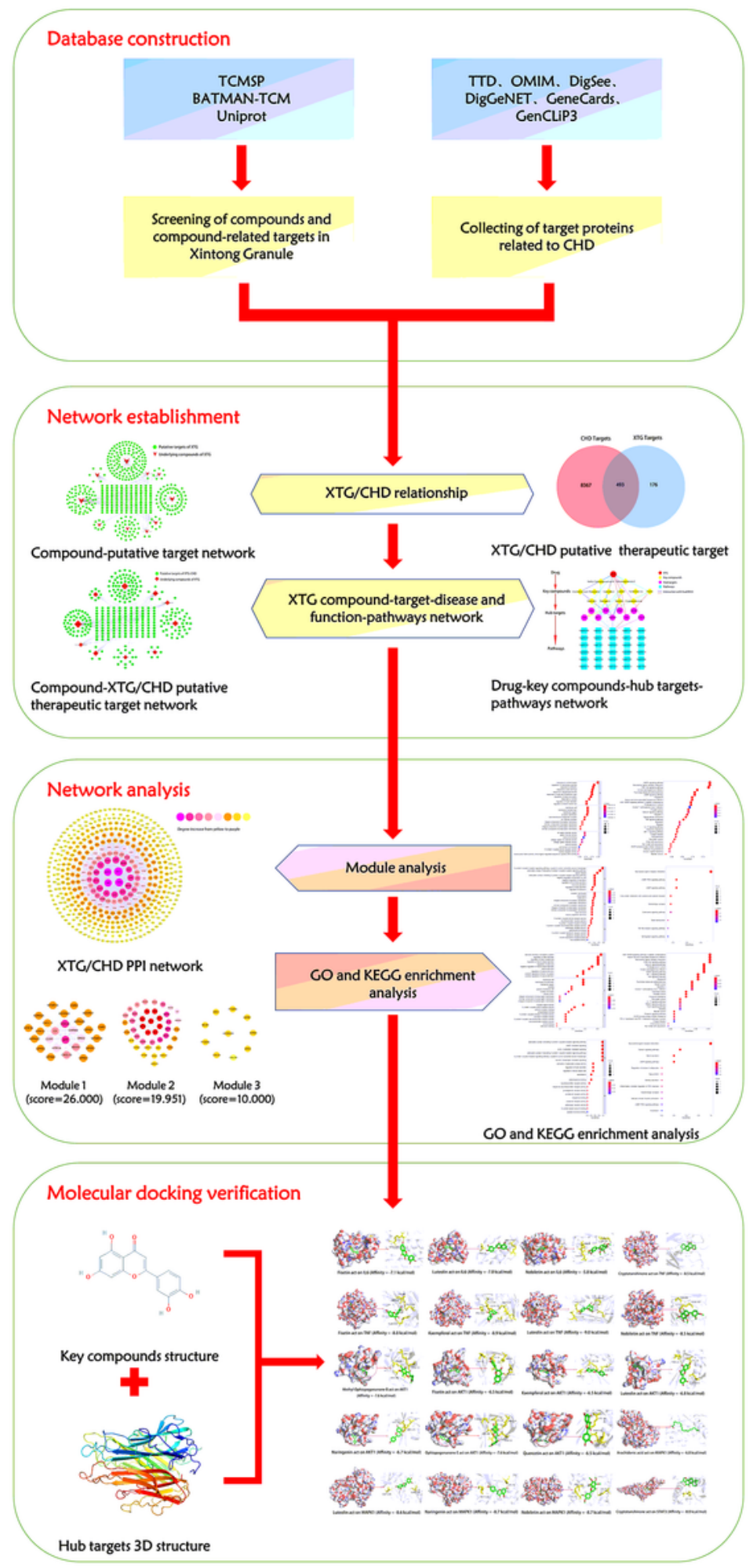

\section{Figure 1}

Network pharmacology and molecular docking verification workflow of XTG for the treatment of CHD. 


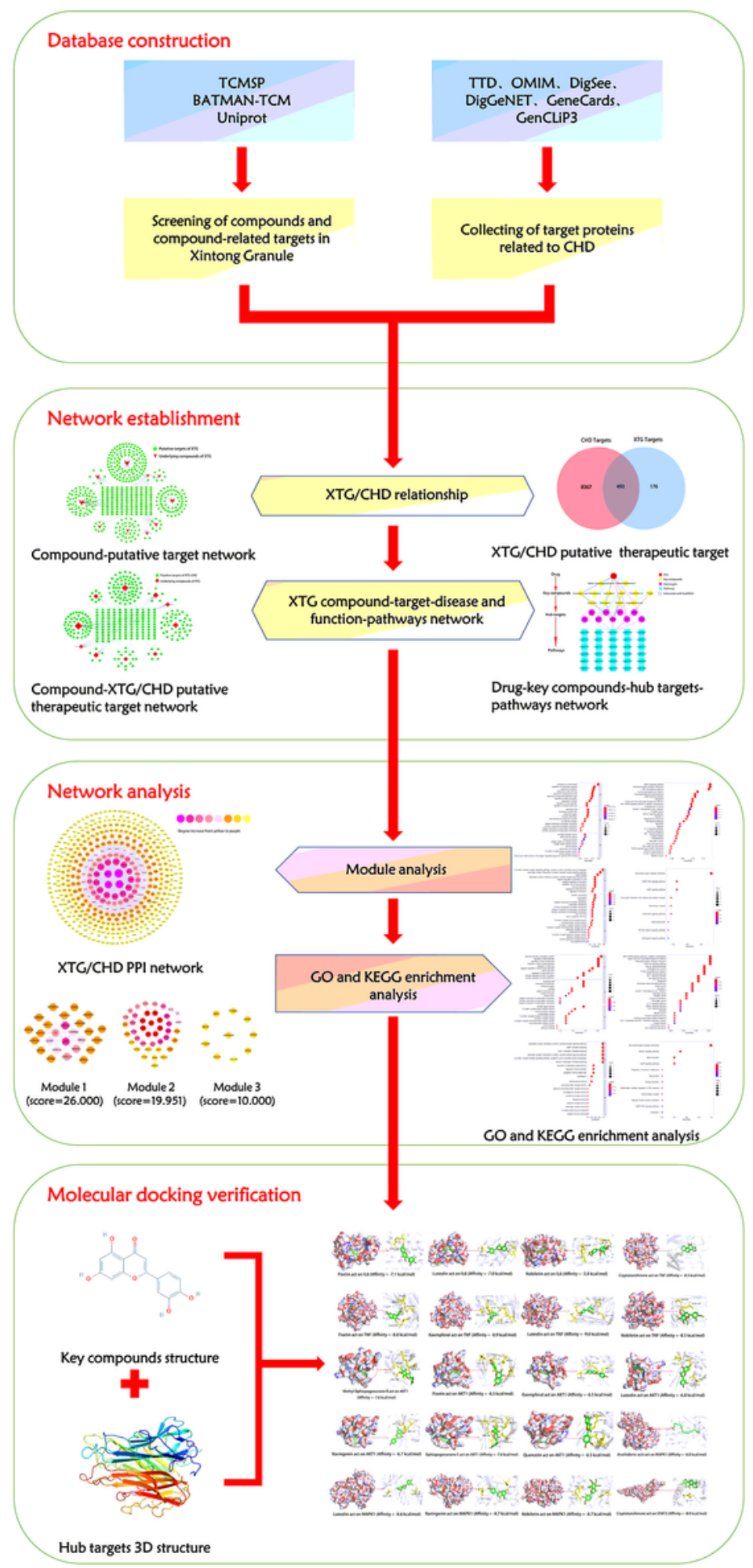

\section{Figure 1}

Network pharmacology and molecular docking verification workflow of XTG for the treatment of CHD. 


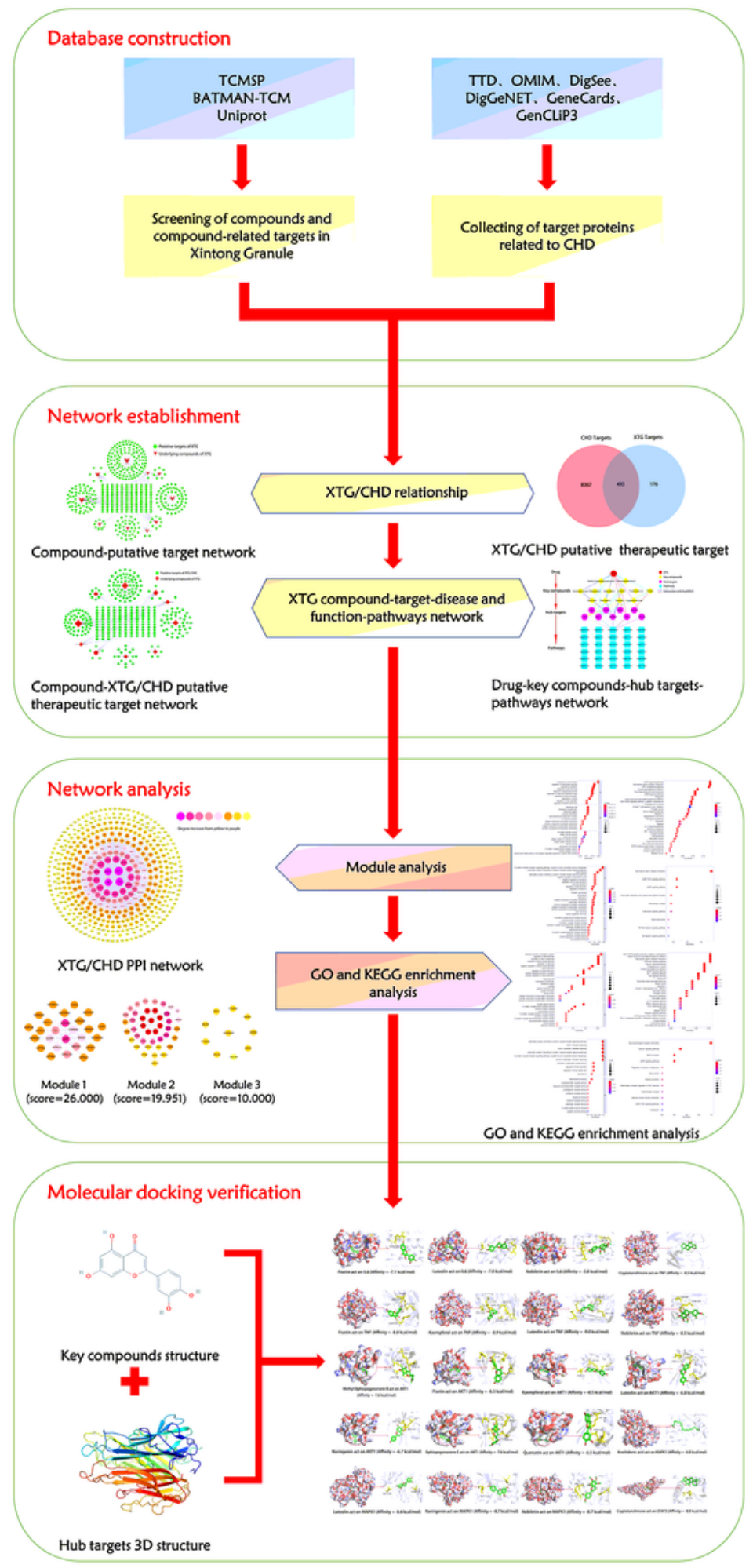

\section{Figure 1}

Network pharmacology and molecular docking verification workflow of XTG for the treatment of CHD. 


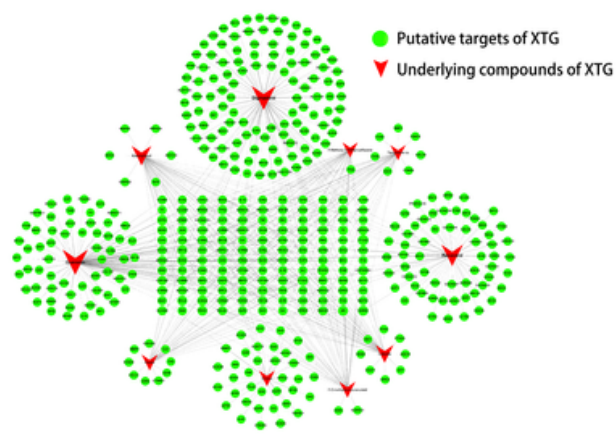

(a)

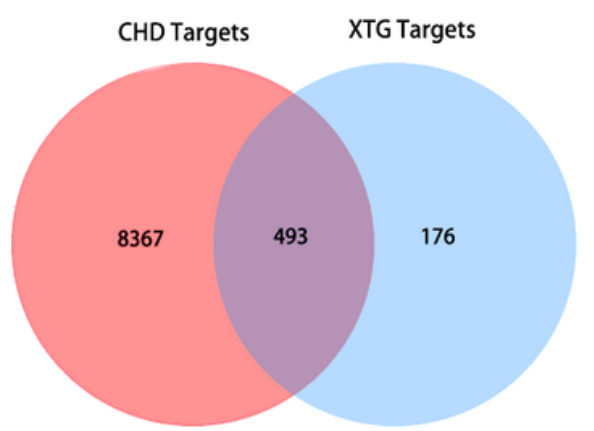

(b)

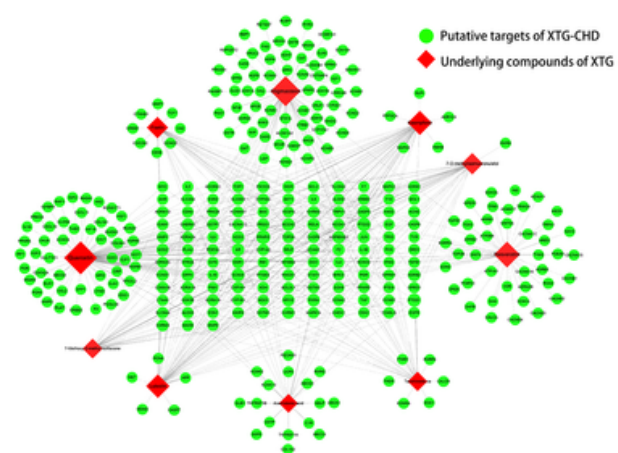

(c)

\section{Figure 2}

Analysis of the active compounds of XTG and putative XTG-CHD targets. (a) Compound-predicted target network of XTG. (b)(c) Compound-XTG/CHD putative therapeutic target network plotting. The size of the nodes is directly proportional to the degree of the nodes.

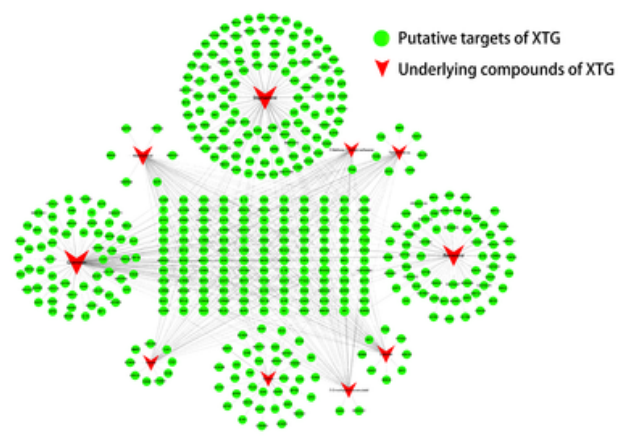

(a)

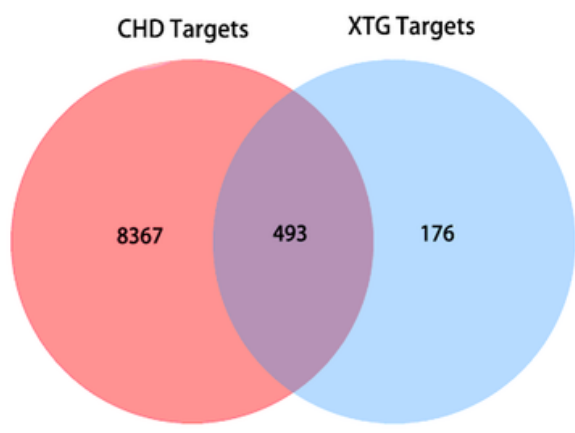

(b)

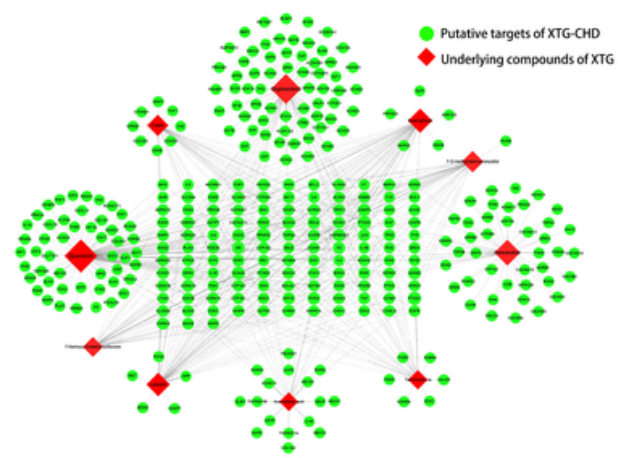

(c)

\section{Figure 2}

Analysis of the active compounds of XTG and putative XTG-CHD targets. (a) Compound-predicted target network of XTG. (b)(c) Compound-XTG/CHD putative therapeutic target network plotting. The size of the nodes is directly proportional to the degree of the nodes.

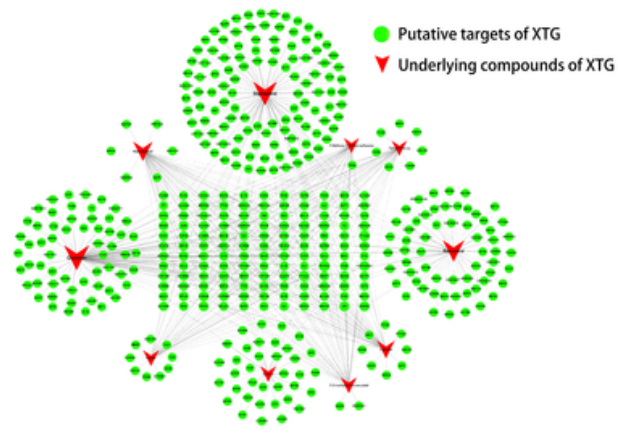

(a)

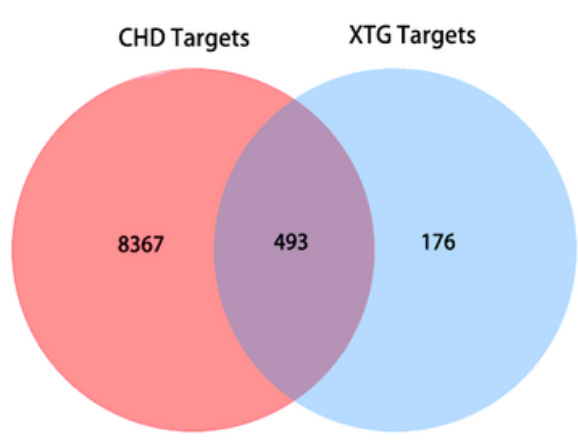

(b)

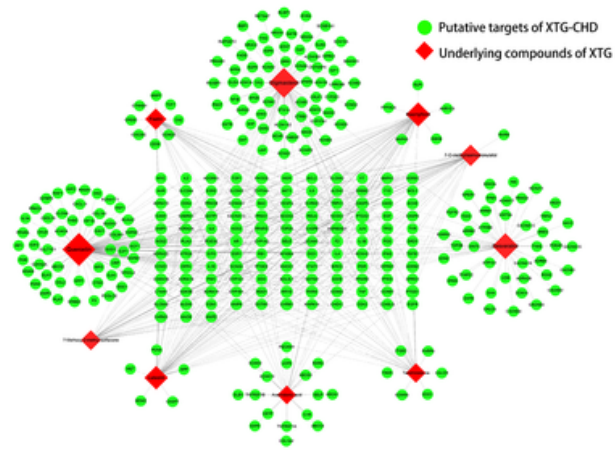

(c)

Figure 2

Analysis of the active compounds of XTG and putative XTG-CHD targets. (a) Compound-predicted target network of XTG. (b)(c) Compound-XTG/CHD putative therapeutic target network plotting. The size of the 
nodes is directly proportional to the degree of the nodes.

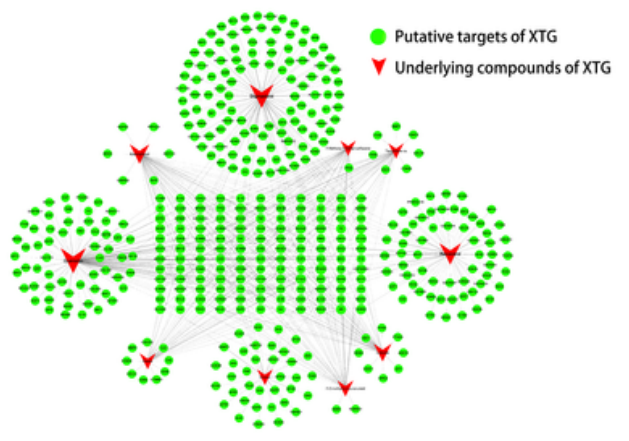

(a)

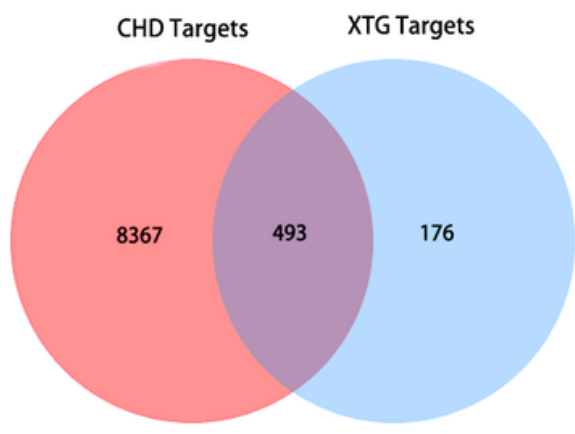

(b)

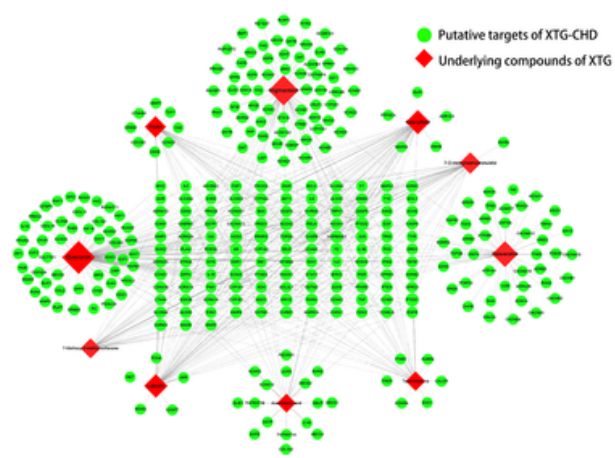

(c)

\section{Figure 2}

Analysis of the active compounds of XTG and putative XTG-CHD targets. (a) Compound-predicted target network of XTG. (b)(c) Compound-XTG/CHD putative therapeutic target network plotting. The size of the nodes is directly proportional to the degree of the nodes. 


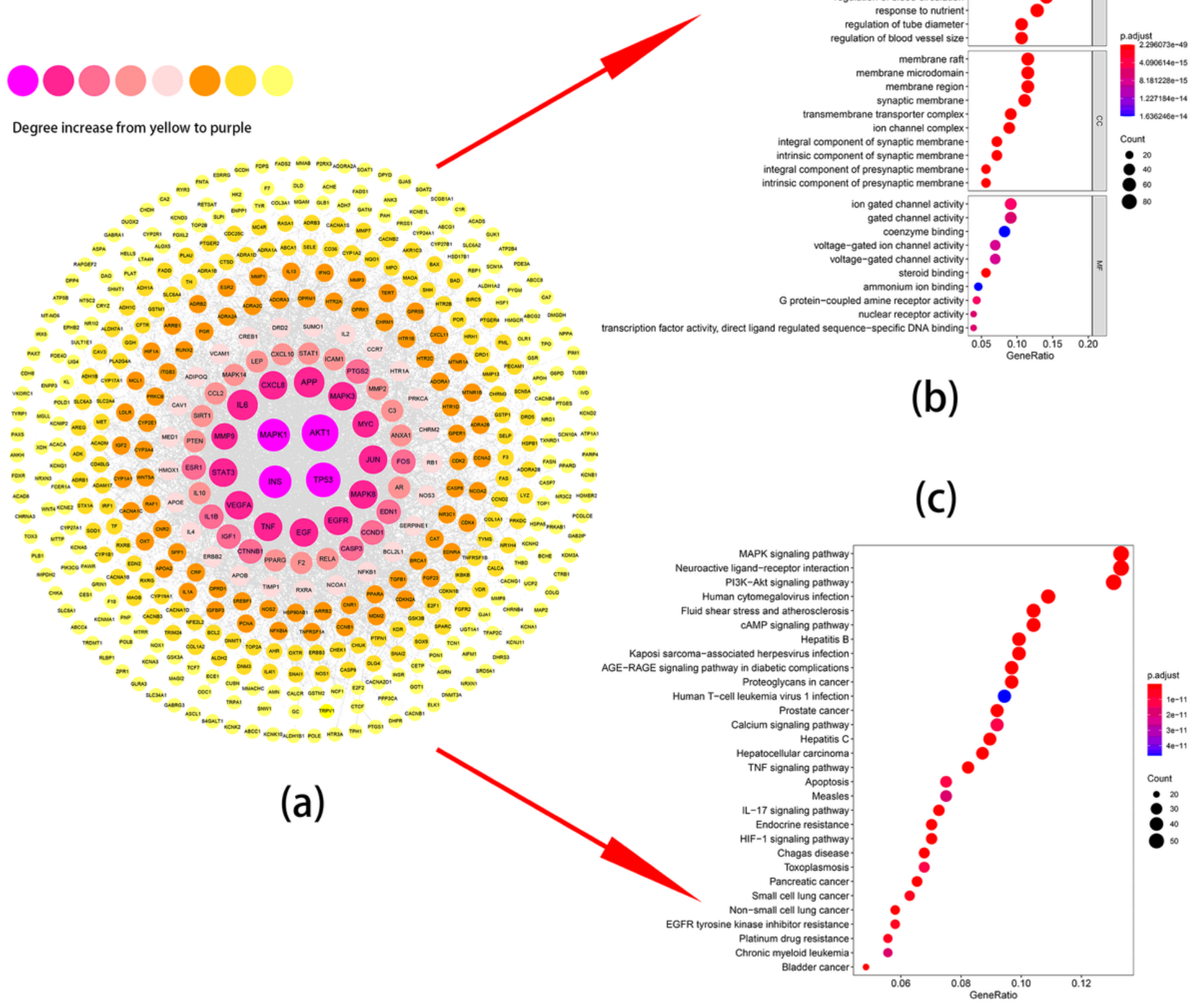

\section{Figure 3}

Analysis of XTG-CHD PPI network and functional enrichment analysis. (a) XTG-CHD PPI network plotting. From yellow to purple, the degree was increasing, and thicker edges denoted the stronger interactions. The size of the nodes is directly proportional to the degree of the nodes. (b) GO enrichment analysis of XTG-CHD PPI network. p-value $<0.01$ and q-value $<0.05$. (c) KEGG pathway analysis of XTG-CHD PPI network. $p$-value $<0.05$ and $q-v a l u e<0.05$. 


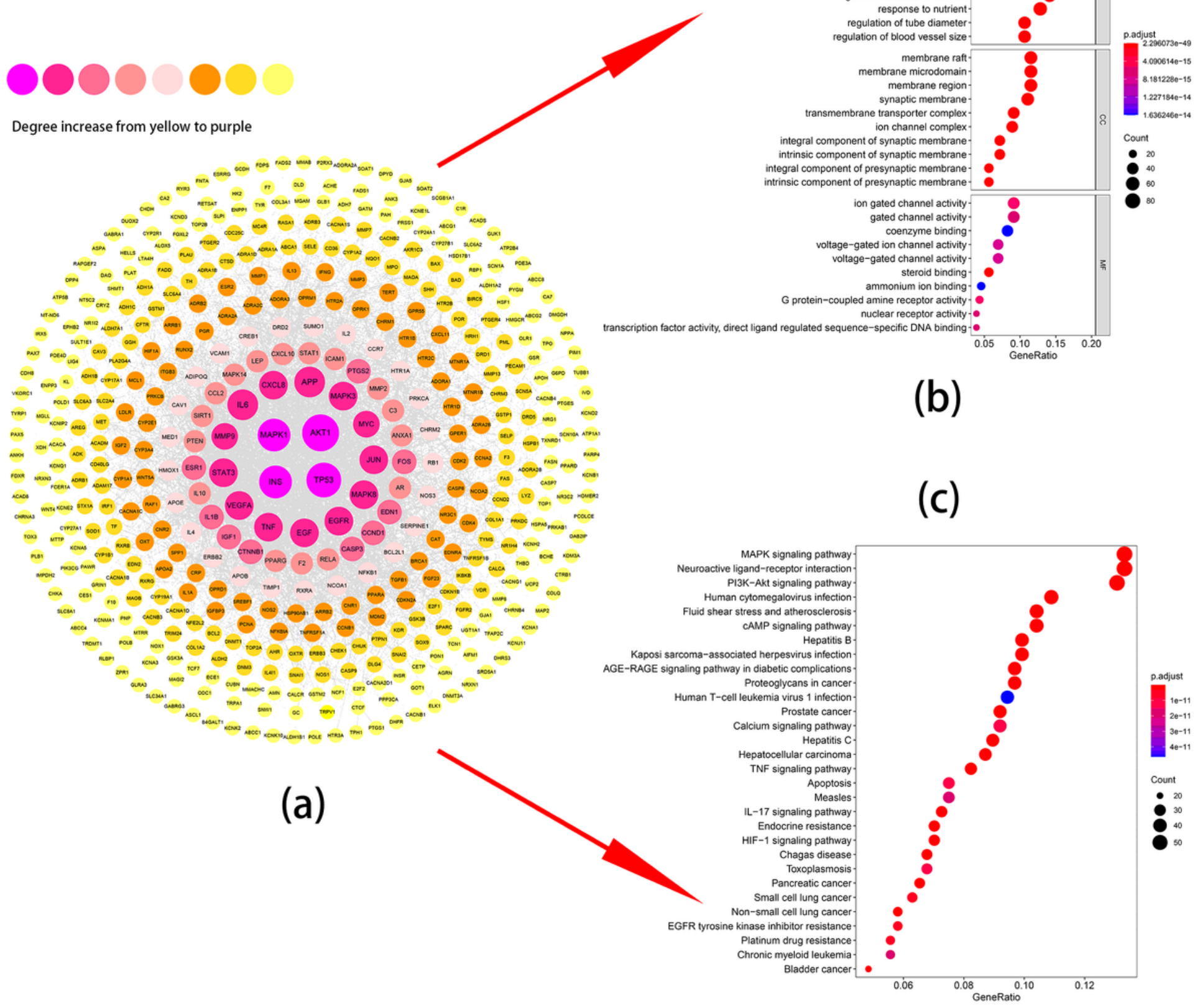

\section{Figure 3}

Analysis of XTG-CHD PPI network and functional enrichment analysis. (a) XTG-CHD PPI network plotting. From yellow to purple, the degree was increasing, and thicker edges denoted the stronger interactions. The size of the nodes is directly proportional to the degree of the nodes. (b) GO enrichment analysis of XTG-CHD PPI network. p-value $<0.01$ and q-value $<0.05$. (c) KEGG pathway analysis of XTG-CHD PPI network. $p$-value $<0.05$ and $q-v a l u e<0.05$. 


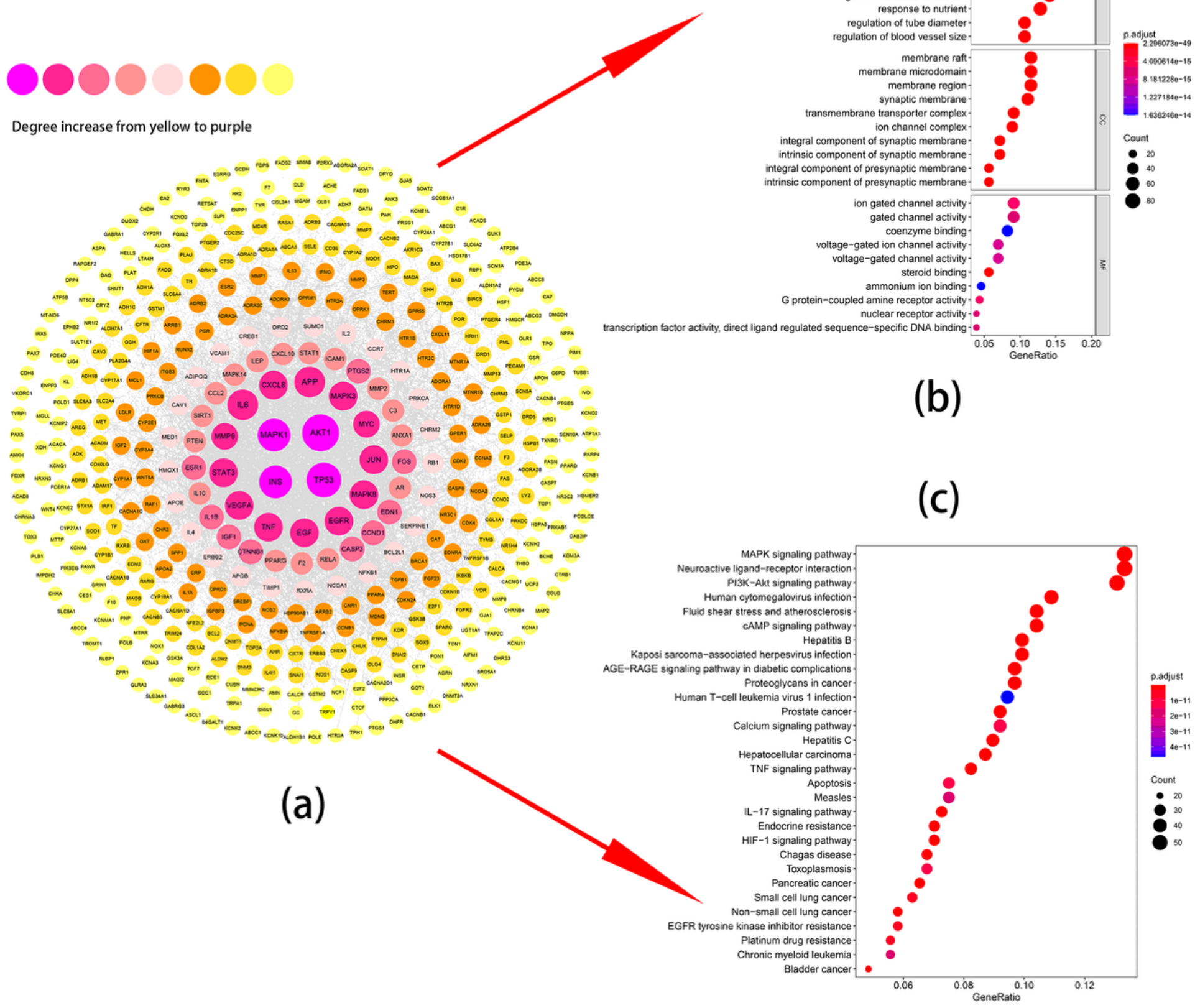

\section{Figure 3}

Analysis of XTG-CHD PPI network and functional enrichment analysis. (a) XTG-CHD PPI network plotting. From yellow to purple, the degree was increasing, and thicker edges denoted the stronger interactions. The size of the nodes is directly proportional to the degree of the nodes. (b) GO enrichment analysis of XTG-CHD PPI network. p-value $<0.01$ and q-value $<0.05$. (c) KEGG pathway analysis of XTG-CHD PPI network. $p$-value $<0.05$ and $q-v a l u e<0.05$. 


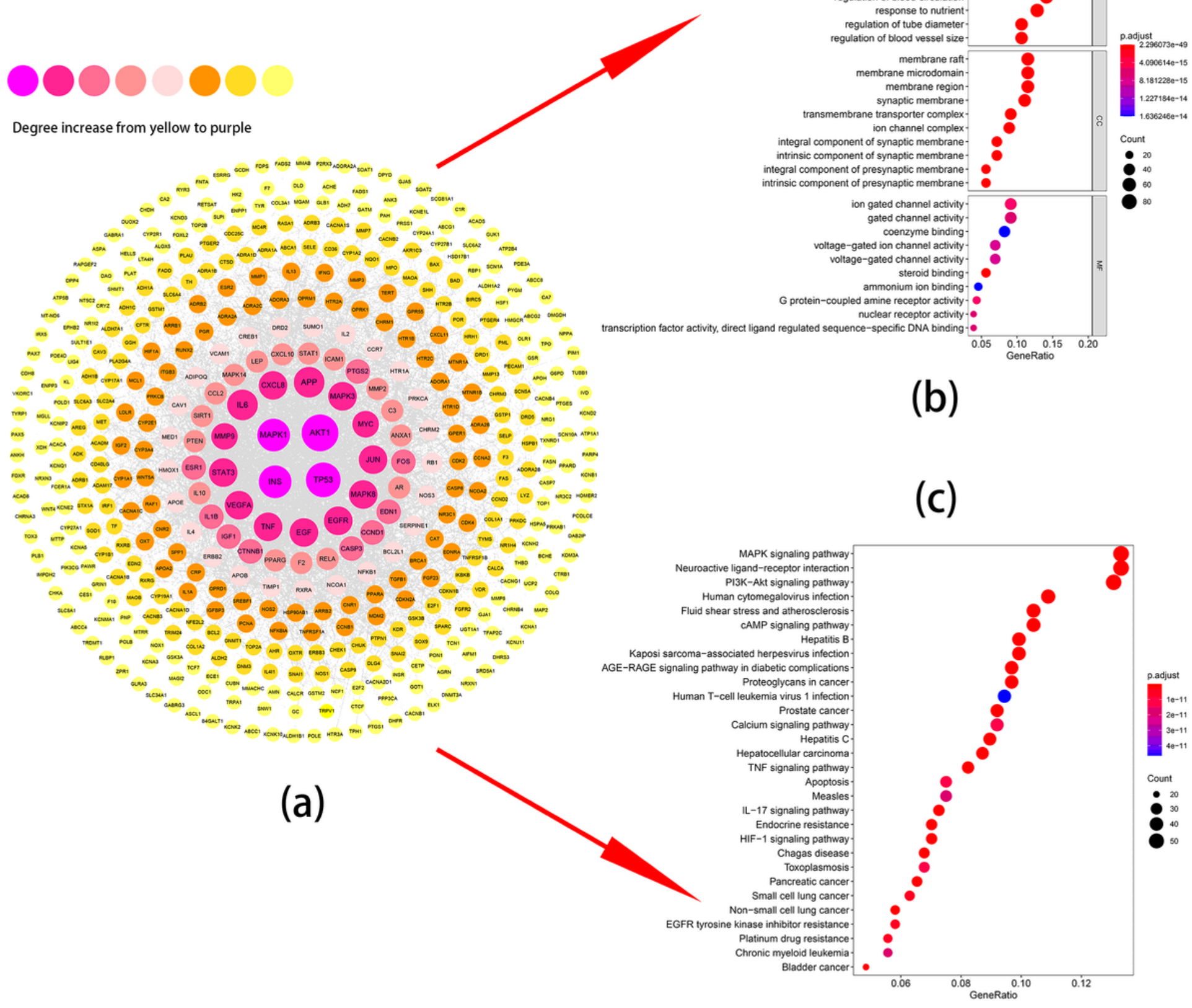

\section{Figure 3}

Analysis of XTG-CHD PPI network and functional enrichment analysis. (a) XTG-CHD PPI network plotting. From yellow to purple, the degree was increasing, and thicker edges denoted the stronger interactions. The size of the nodes is directly proportional to the degree of the nodes. (b) GO enrichment analysis of XTG-CHD PPI network. p-value $<0.01$ and q-value $<0.05$. (c) KEGG pathway analysis of XTG-CHD PPI network. $p$-value $<0.05$ and $q-v a l u e<0.05$. 


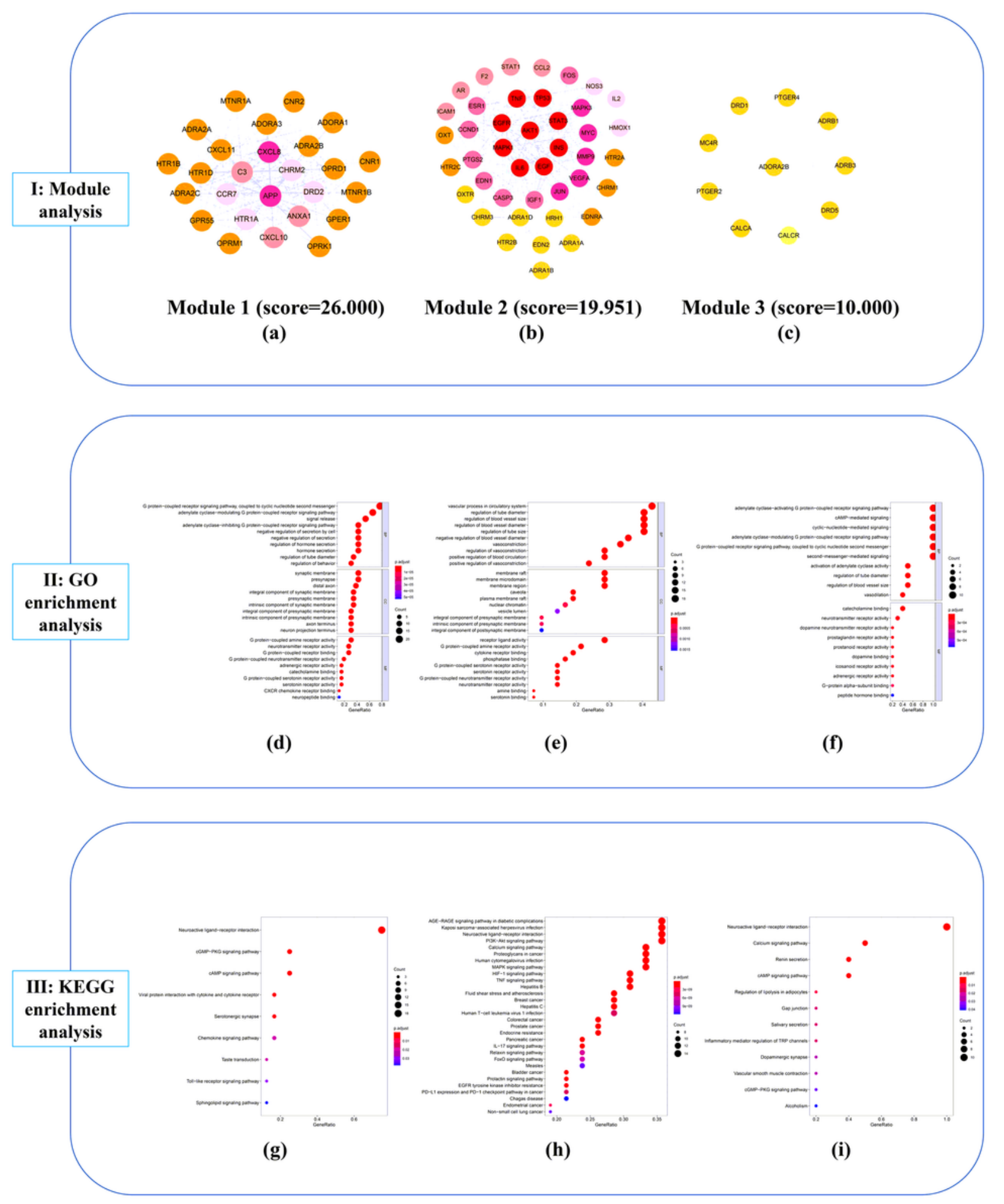

\section{Figure 4}

Analysis of clusters and functional enrichment analysis. I: Clusters of the XTG-CHD PPI network. (a) Module 1 (score $=26.000)$. (b) Module 2 (score $=19.951)$. (c) Module 3 (score $=10.000)$. Red circles represent XTG-CHD hub targets, and the remaining circles represent other human protein targets associated with disease treatment targets. II: GO enrichment analysis for each cluster. The top $10 \mathrm{GO}$ terms were shown in the figure; $\mathrm{p}$-value $<0.01$ and q-value < 0.05; (d) GO enrichment analysis of Module 
1. (e) GO enrichment analysis of Module 2. (f) GO enrichment analysis of Module 3. III: KEGG pathway enrichment analysis for each cluster. p-value < 0.05 and q-value < 0.05; (g): KEGG pathway analysis of Module 1. (h) KEGG pathway analysis of Module 2. (i) KEGG pathway analysis of Module 3. The top 30 KEGG pathways were shown in the figure. The $y$-axis shows significantly enriched KEGG pathways, and $x$ axis shows the gene counts.

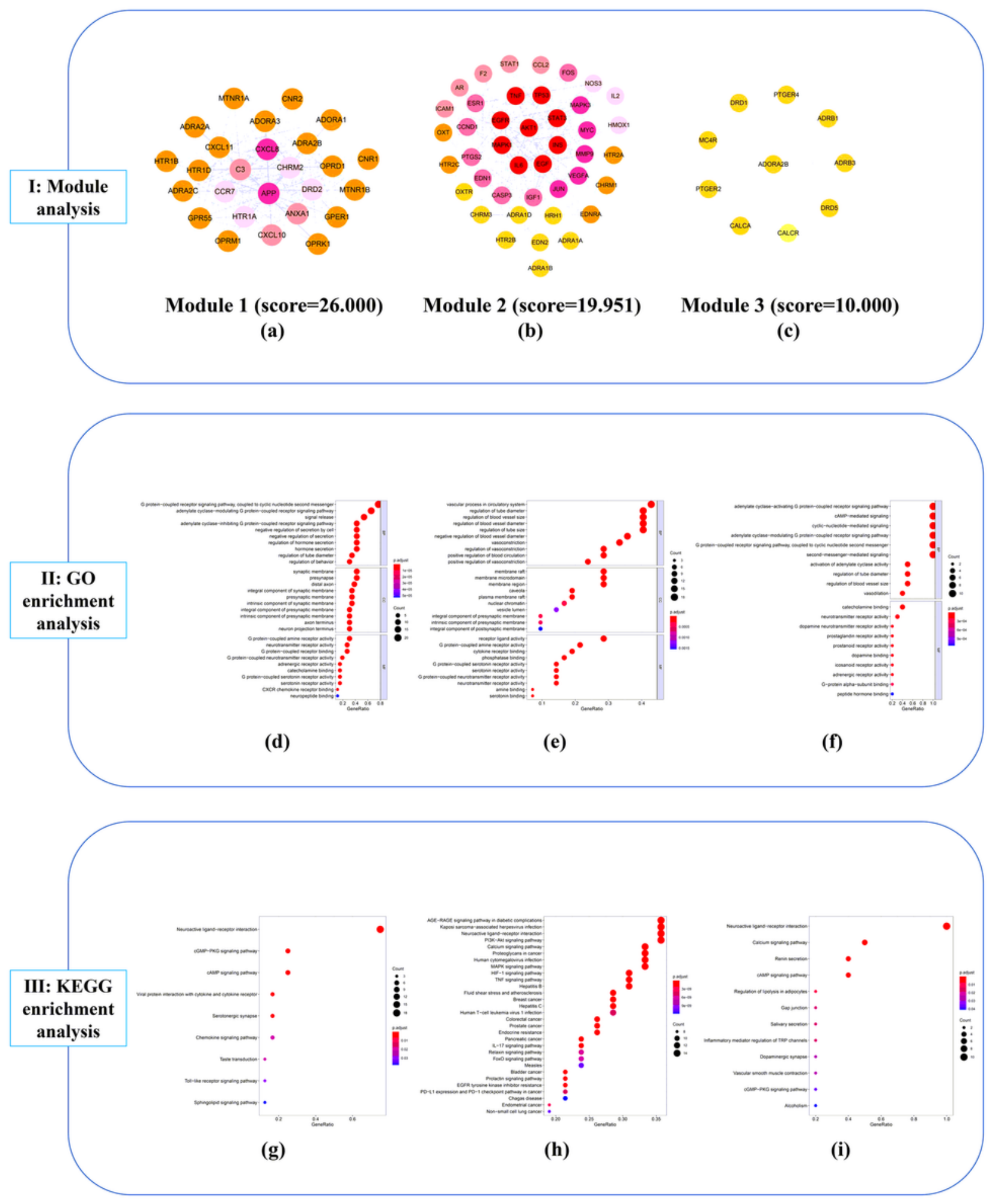

Figure 4 
Analysis of clusters and functional enrichment analysis. I: Clusters of the XTG-CHD PPI network. (a) Module 1 (score $=26.000)$. (b) Module 2 (score $=19.951)$. (c) Module 3 (score $=10.000)$. Red circles represent XTG-CHD hub targets, and the remaining circles represent other human protein targets associated with disease treatment targets. II: GO enrichment analysis for each cluster. The top $10 \mathrm{GO}$ terms were shown in the figure; $p$-value $<0.01$ and q-value $<0.05$; (d) GO enrichment analysis of Module 1. (e) GO enrichment analysis of Module 2. (f) GO enrichment analysis of Module 3. III: KEGG pathway enrichment analysis for each cluster. p-value < 0.05 and q-value < 0.05; (g): KEGG pathway analysis of Module 1. (h) KEGG pathway analysis of Module 2. (i) KEGG pathway analysis of Module 3 . The top 30 KEGG pathways were shown in the figure. The $y$-axis shows significantly enriched KEGG pathways, and $x-$ axis shows the gene counts. 

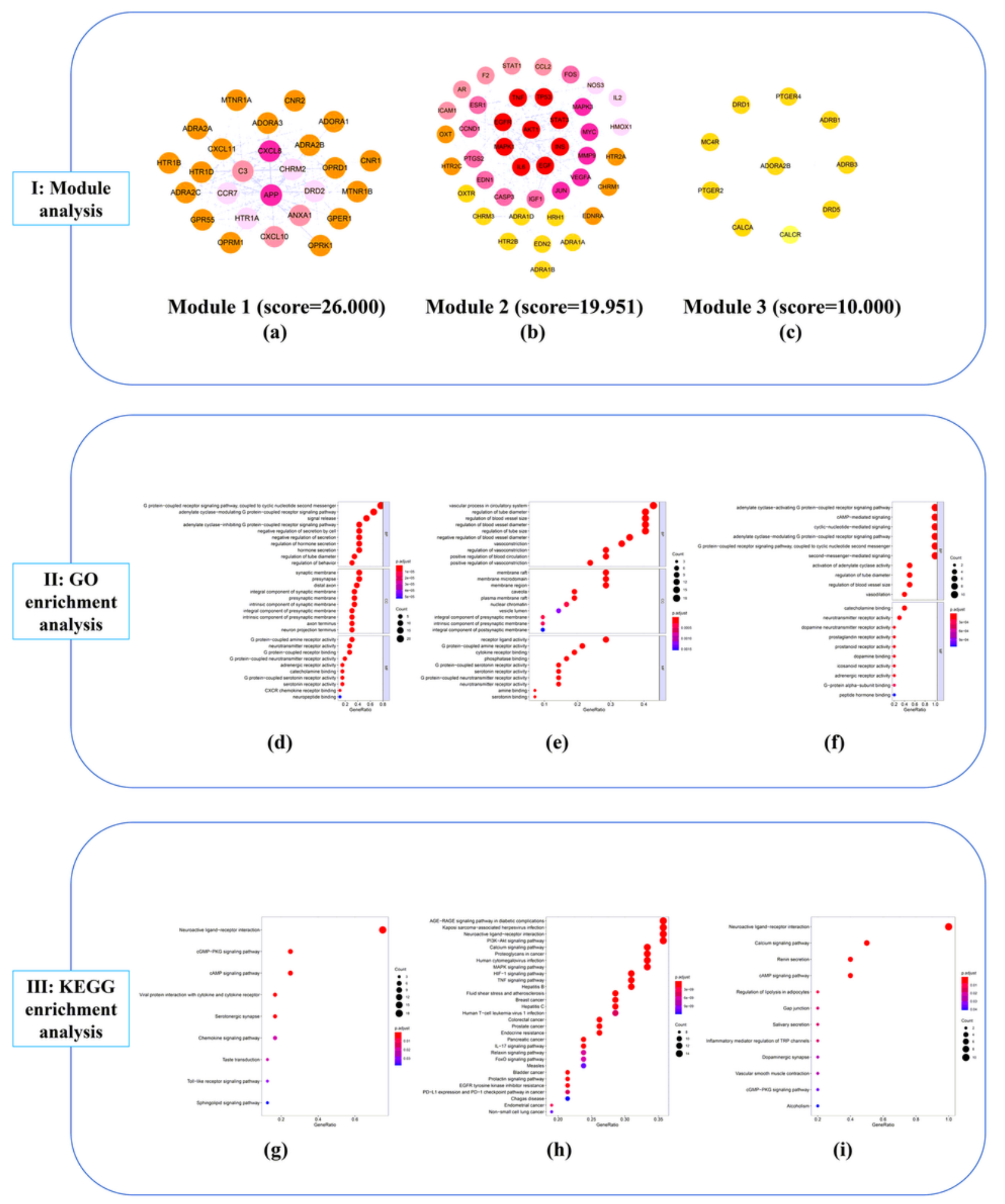

\section{Figure 4}

Analysis of clusters and functional enrichment analysis. I: Clusters of the XTG-CHD PPI network. (a) Module 1 (score $=26.000)$. (b) Module 2 (score $=19.951)$. (c) Module 3 (score $=10.000)$. Red circles represent XTG-CHD hub targets, and the remaining circles represent other human protein targets associated with disease treatment targets. II: GO enrichment analysis for each cluster. The top $10 \mathrm{GO}$ terms were shown in the figure; $p$-value $<0.01$ and q-value < 0.05; (d) GO enrichment analysis of Module 
1. (e) GO enrichment analysis of Module 2. (f) GO enrichment analysis of Module 3. III: KEGG pathway enrichment analysis for each cluster. p-value < 0.05 and q-value < 0.05; (g): KEGG pathway analysis of Module 1. (h) KEGG pathway analysis of Module 2. (i) KEGG pathway analysis of Module 3. The top 30 KEGG pathways were shown in the figure. The $y$-axis shows significantly enriched KEGG pathways, and $x$ axis shows the gene counts.

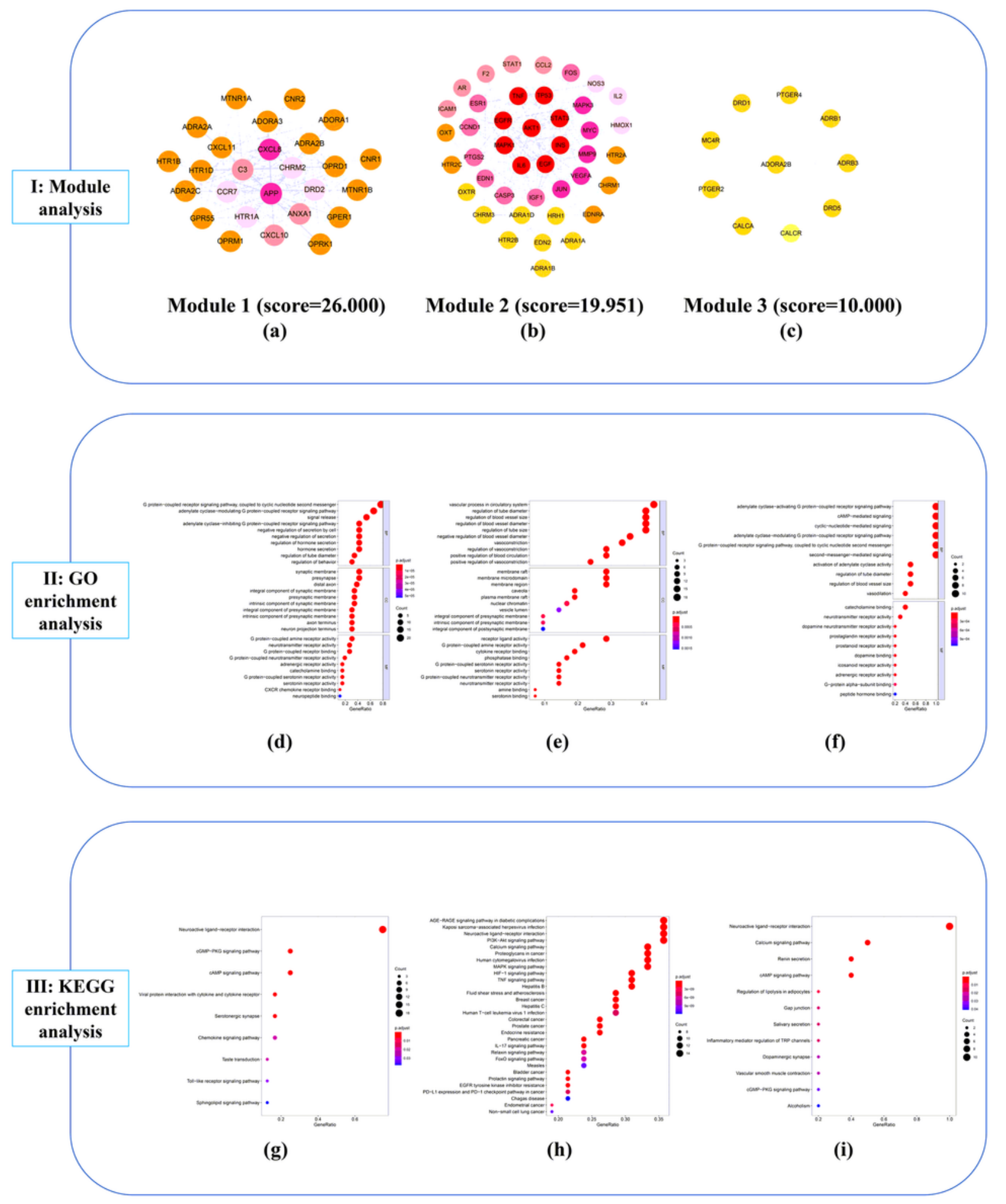

Figure 4 
Analysis of clusters and functional enrichment analysis. I: Clusters of the XTG-CHD PPI network. (a) Module 1 (score $=26.000)$. (b) Module 2 (score $=19.951)$. (c) Module 3 (score $=10.000)$. Red circles represent XTG-CHD hub targets, and the remaining circles represent other human protein targets associated with disease treatment targets. II: GO enrichment analysis for each cluster. The top $10 \mathrm{GO}$ terms were shown in the figure; $p$-value $<0.01$ and q-value $<0.05$; (d) GO enrichment analysis of Module 1. (e) GO enrichment analysis of Module 2. (f) GO enrichment analysis of Module 3. III: KEGG pathway enrichment analysis for each cluster. p-value < 0.05 and q-value < 0.05; (g): KEGG pathway analysis of Module 1. (h) KEGG pathway analysis of Module 2. (i) KEGG pathway analysis of Module 3. The top 30 KEGG pathways were shown in the figure. The $y$-axis shows significantly enriched KEGG pathways, and $x-$ axis shows the gene counts.

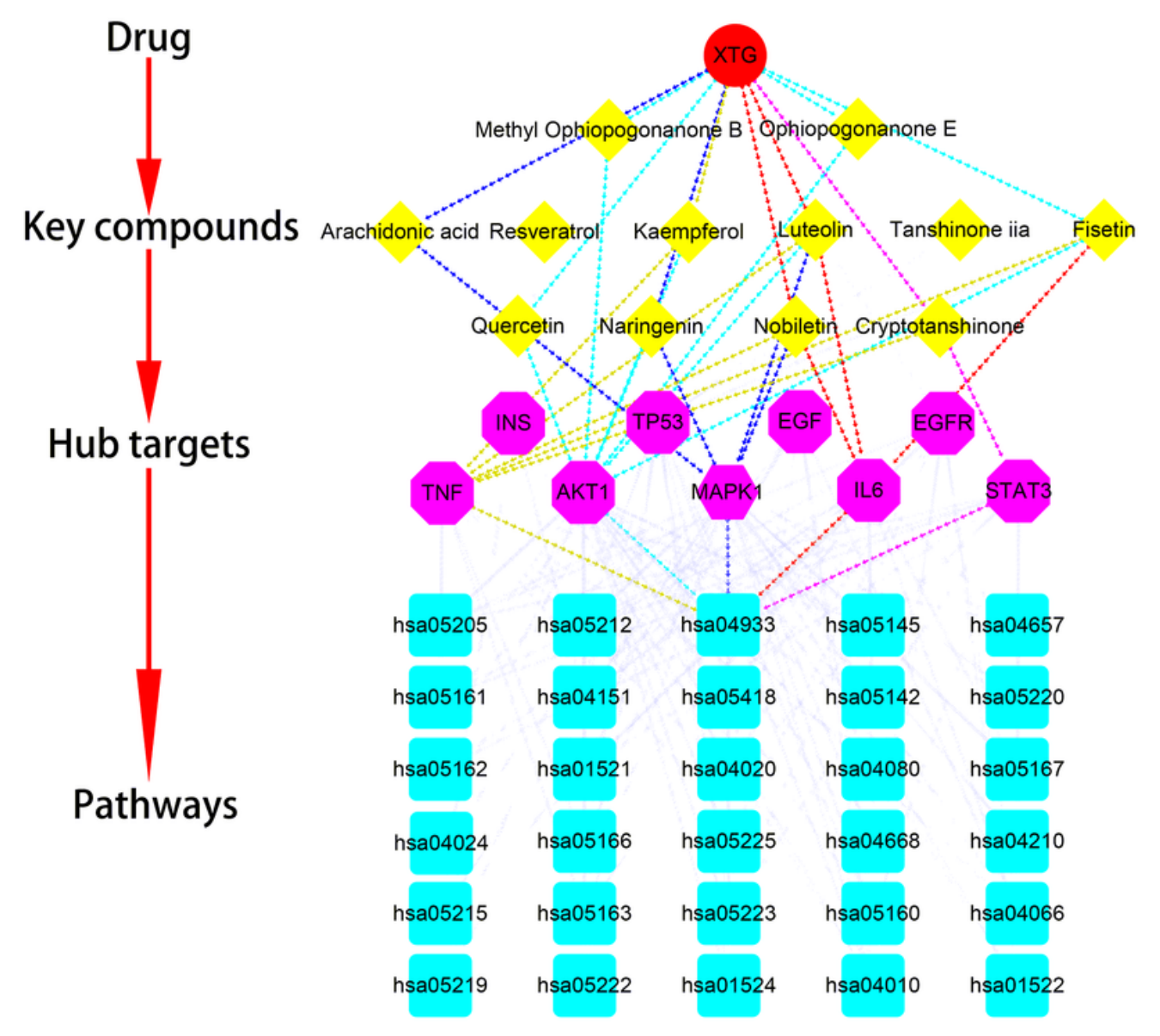

XTG

Key compounds

Hub targets

Pathways

暏整 Interaction with hsa04933

\section{Figure 5}

Drug-key compounds-hub targets-pathways network. 


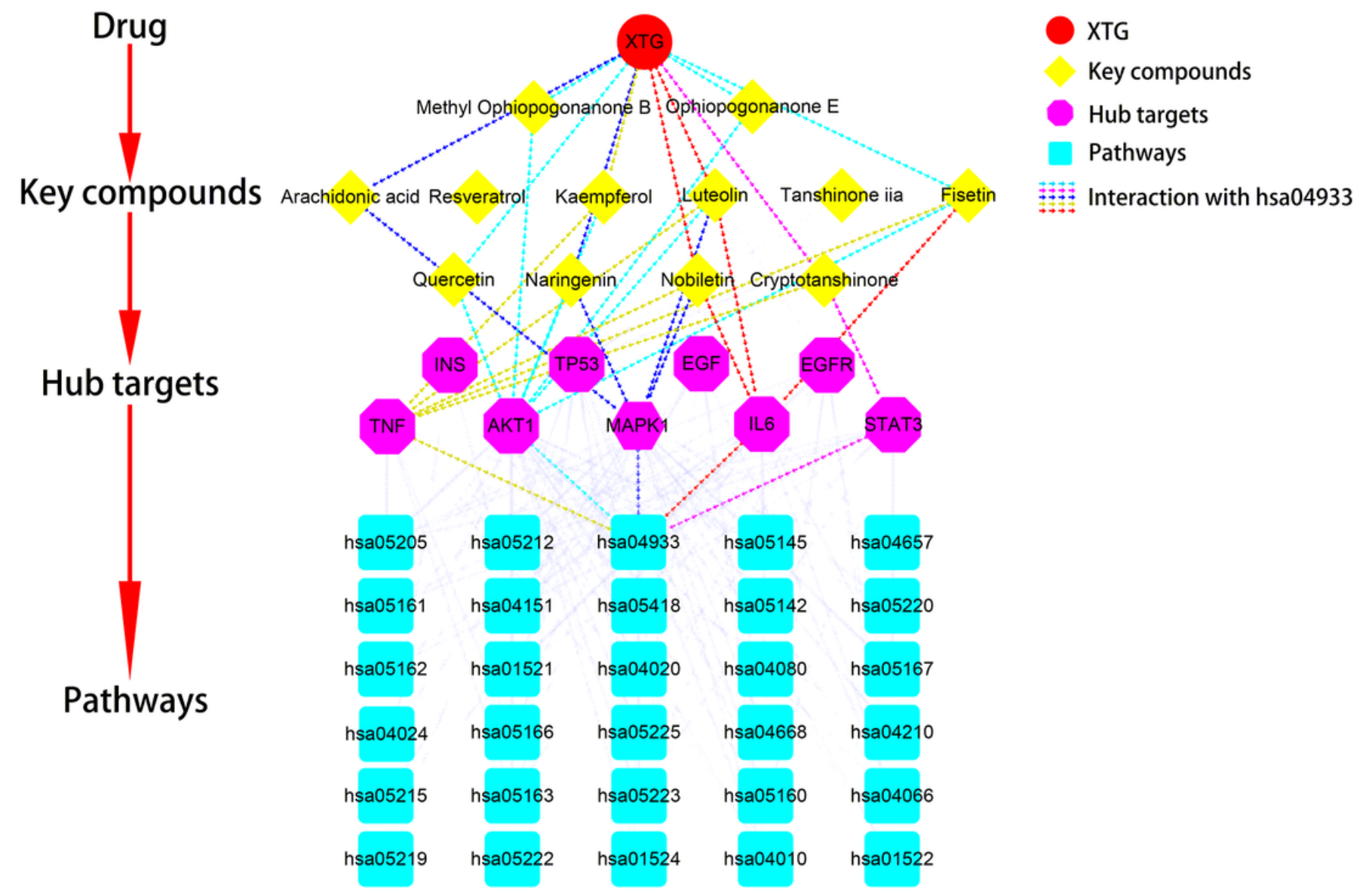

\section{Figure 5}

Drug-key compounds-hub targets-pathways network. 


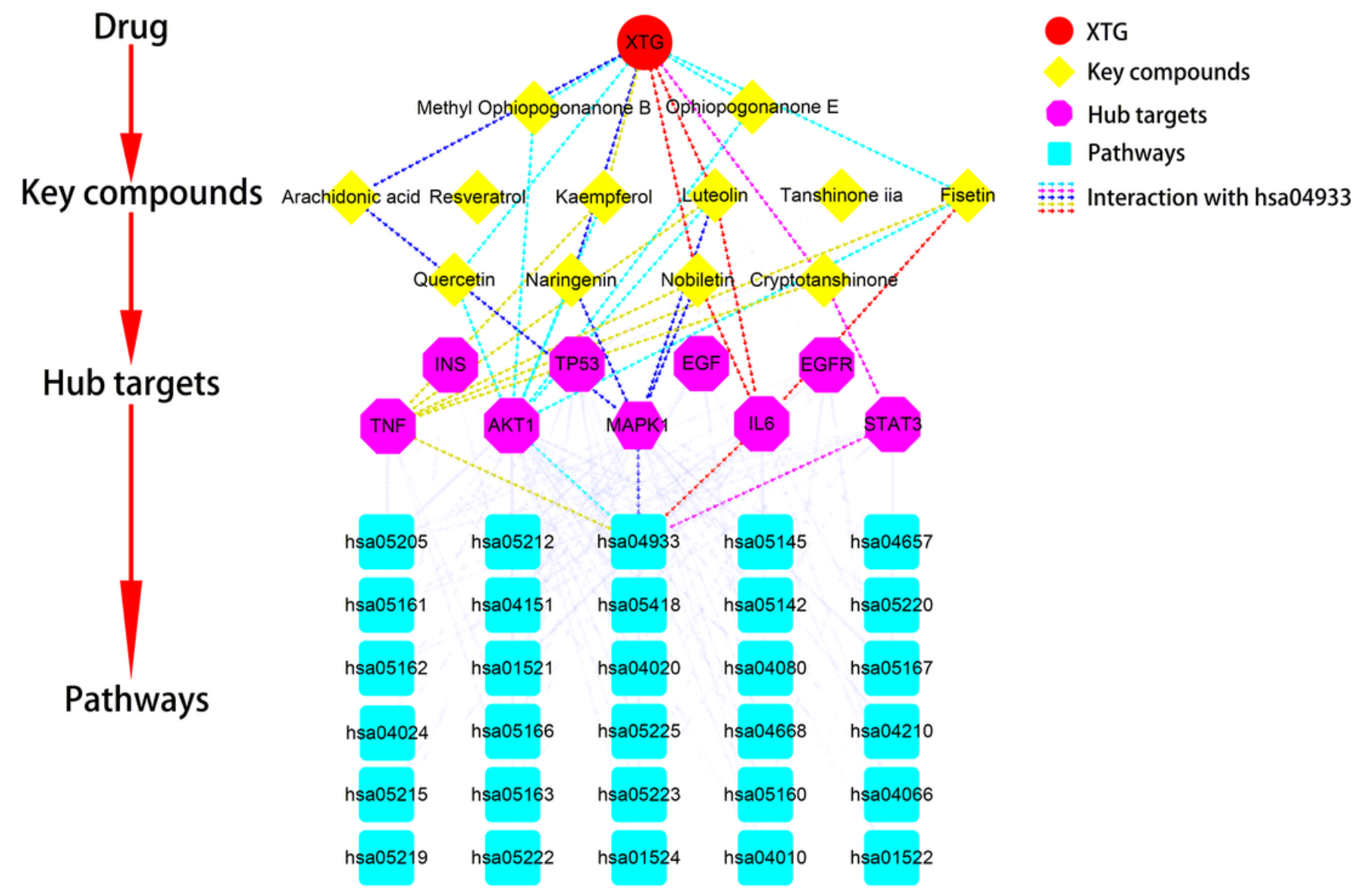

\section{Figure 5}

Drug-key compounds-hub targets-pathways network. 


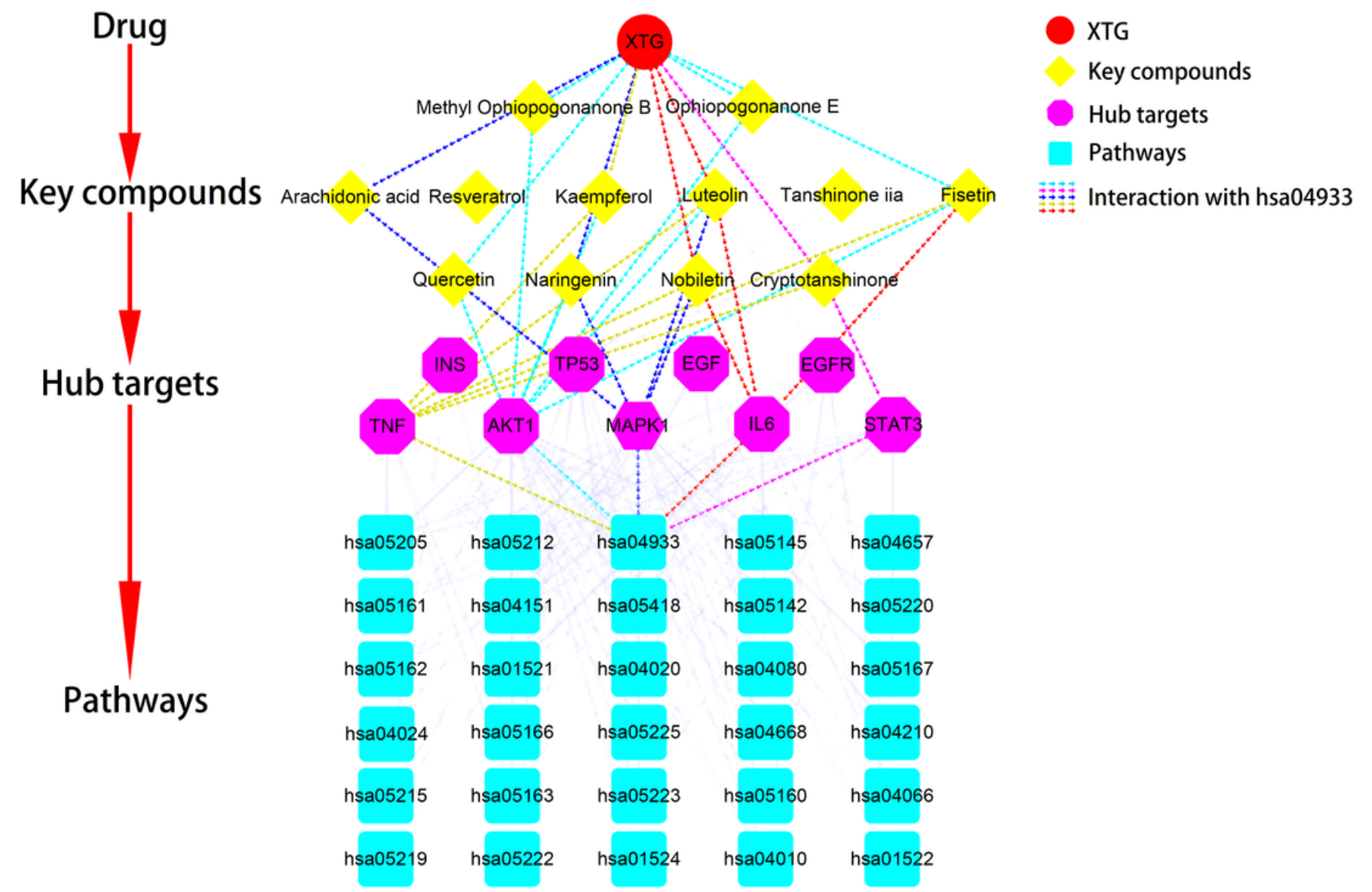

\section{Figure 5}

Drug-key compounds-hub targets-pathways network. 


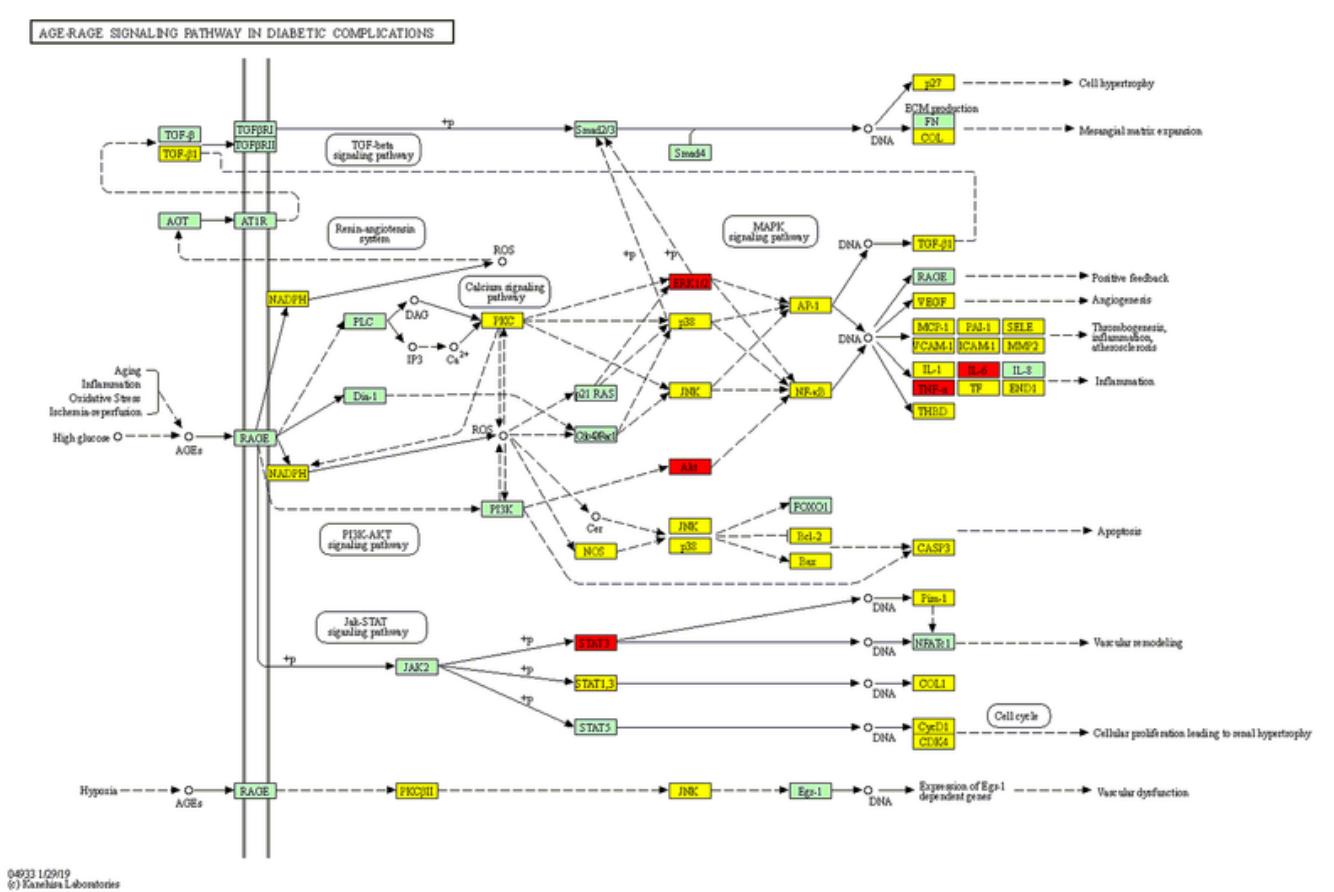

(a)

\section{AGE-RAGE signaling pathway in diabetic complications}

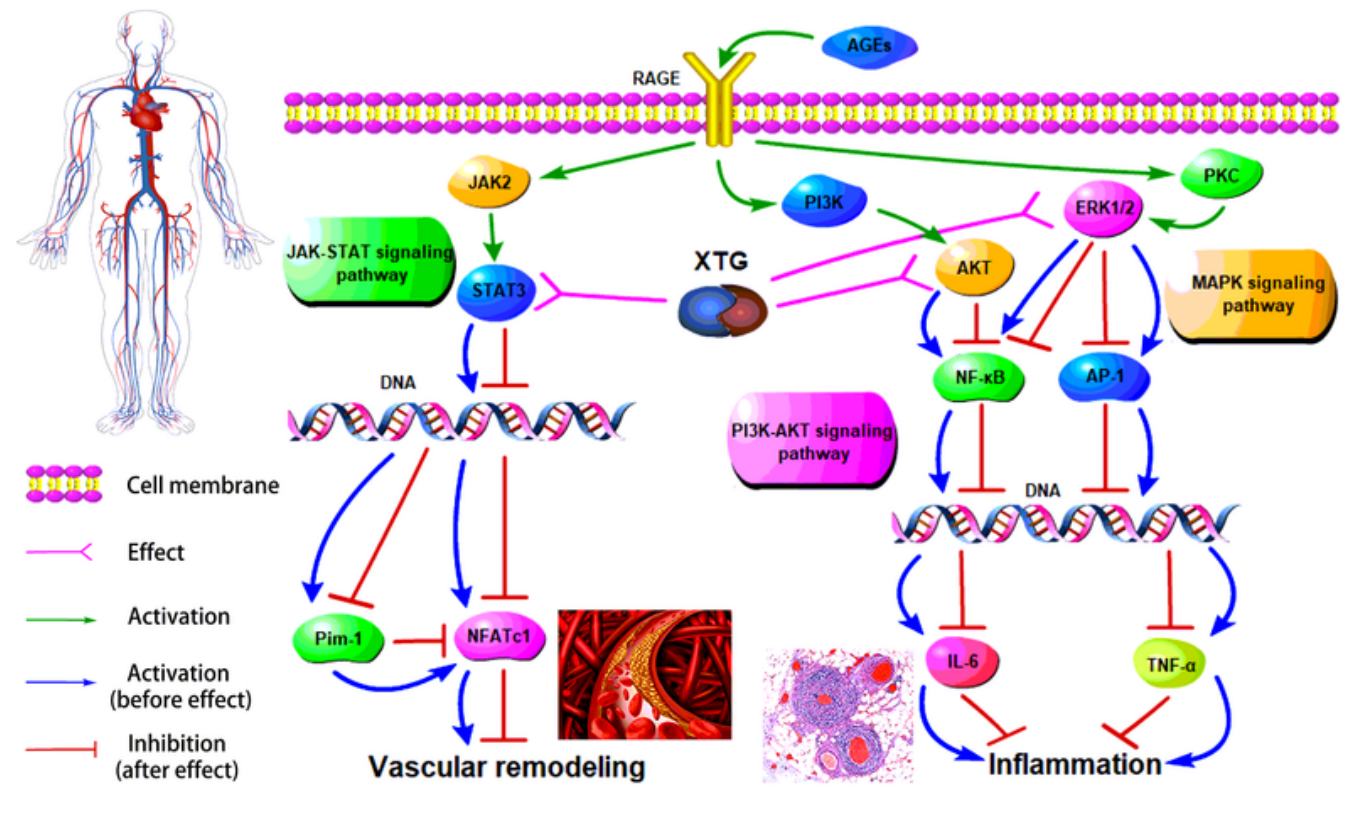

(b)

\section{Figure 6}

(a) Modulating AGE-RAGE signaling pathway in diabetic complications of XTG against CHD. Hub targets were colored in red, targets of XTG-CHD were colored in yellow, and other protein targets in the pathway were colored in green. (b) Illustration of the crucial biological processes involving putative targets and known therapeutic targets of XTG: Signal transducer and activator of transcription 3 (STAT3), RAC-alpha 
serine/threonine-protein kinase (AKT1), Mitogen-activated protein kinase 1 (MAPK1), Tumor necrosis factor (TNF), Interleukin-6 (IL6).

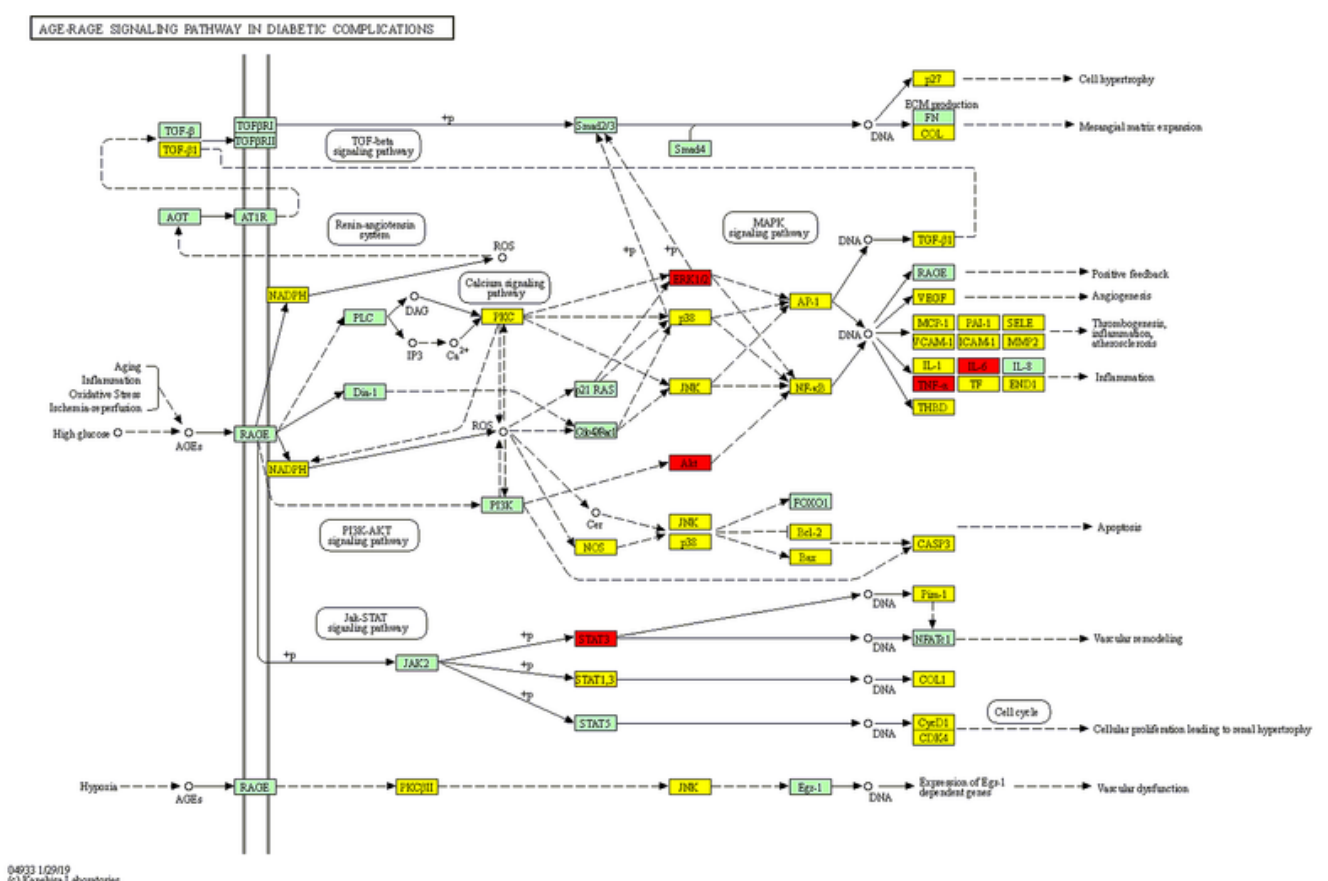

(a)

\section{AGE-RAGE signaling pathway in diabetic complications}

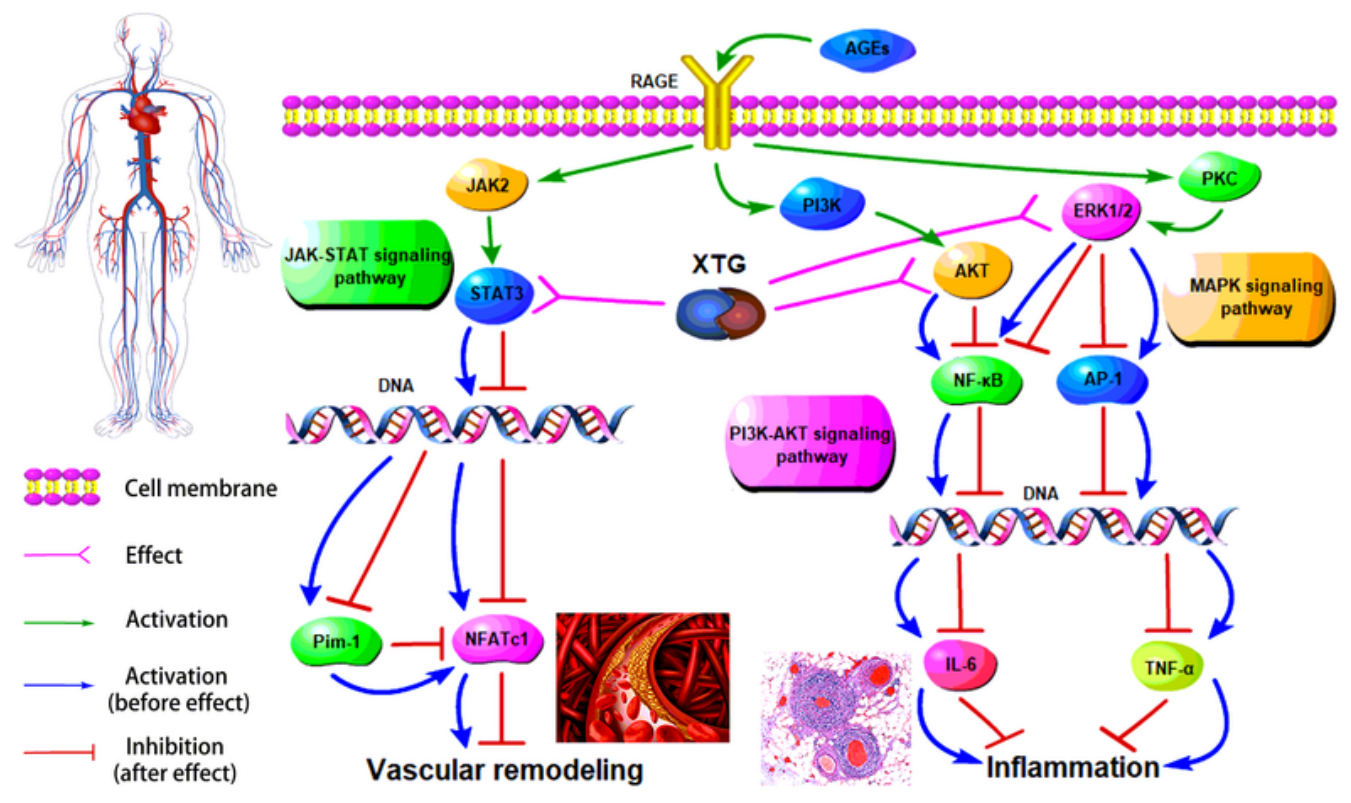

(b)

Figure 6

(a) Modulating AGE-RAGE signaling pathway in diabetic complications of XTG against CHD. Hub targets were colored in red, targets of XTG-CHD were colored in yellow, and other protein targets in the pathway were colored in green. (b) Illustration of the crucial biological processes involving putative targets and 
known therapeutic targets of XTG: Signal transducer and activator of transcription 3 (STAT3), RAC-alpha serine/threonine-protein kinase (AKT1), Mitogen-activated protein kinase 1 (MAPK1), Tumor necrosis factor (TNF), Interleukin-6 (IL6).

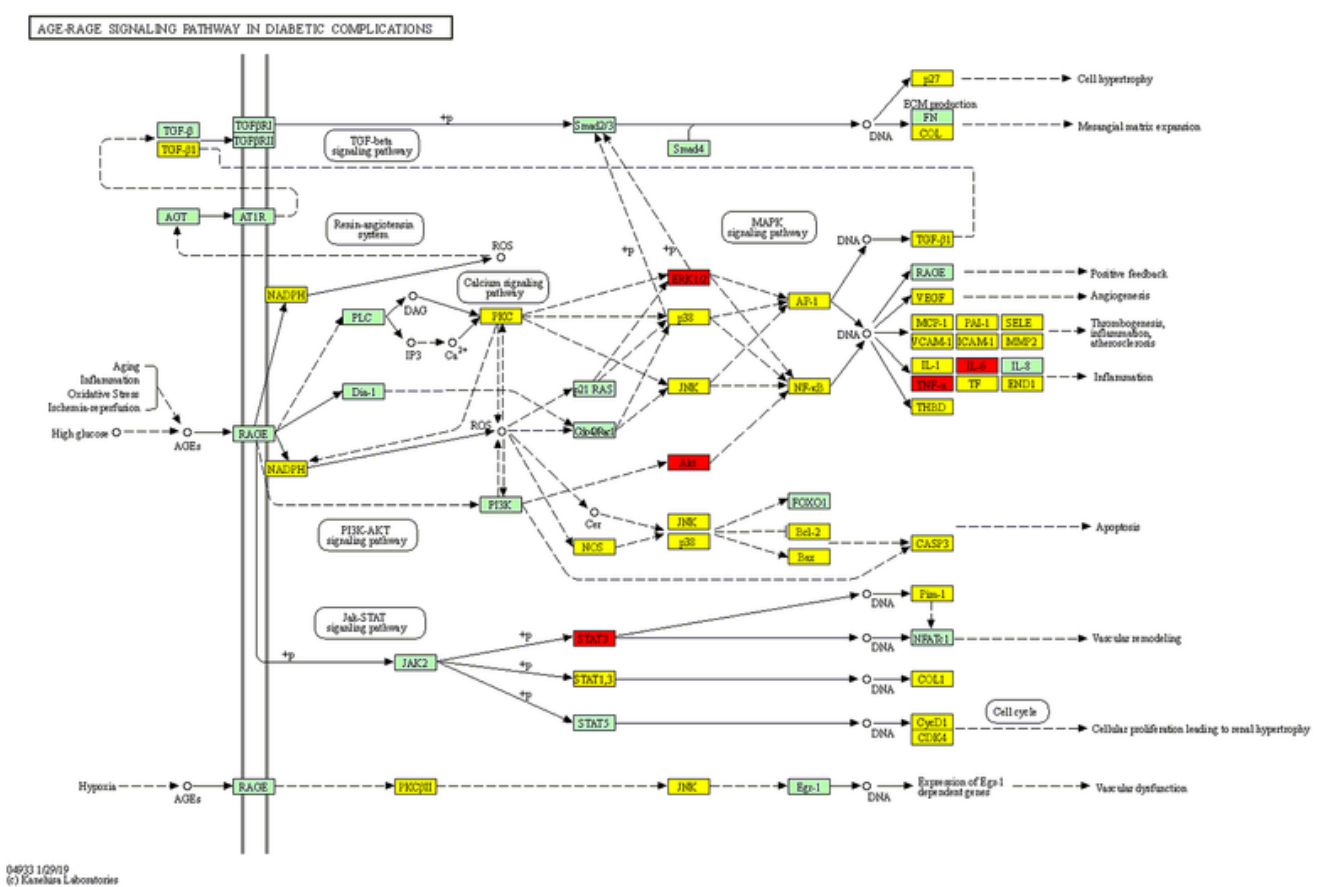

(a)

\section{AGE-RAGE signaling pathway in diabetic complications}

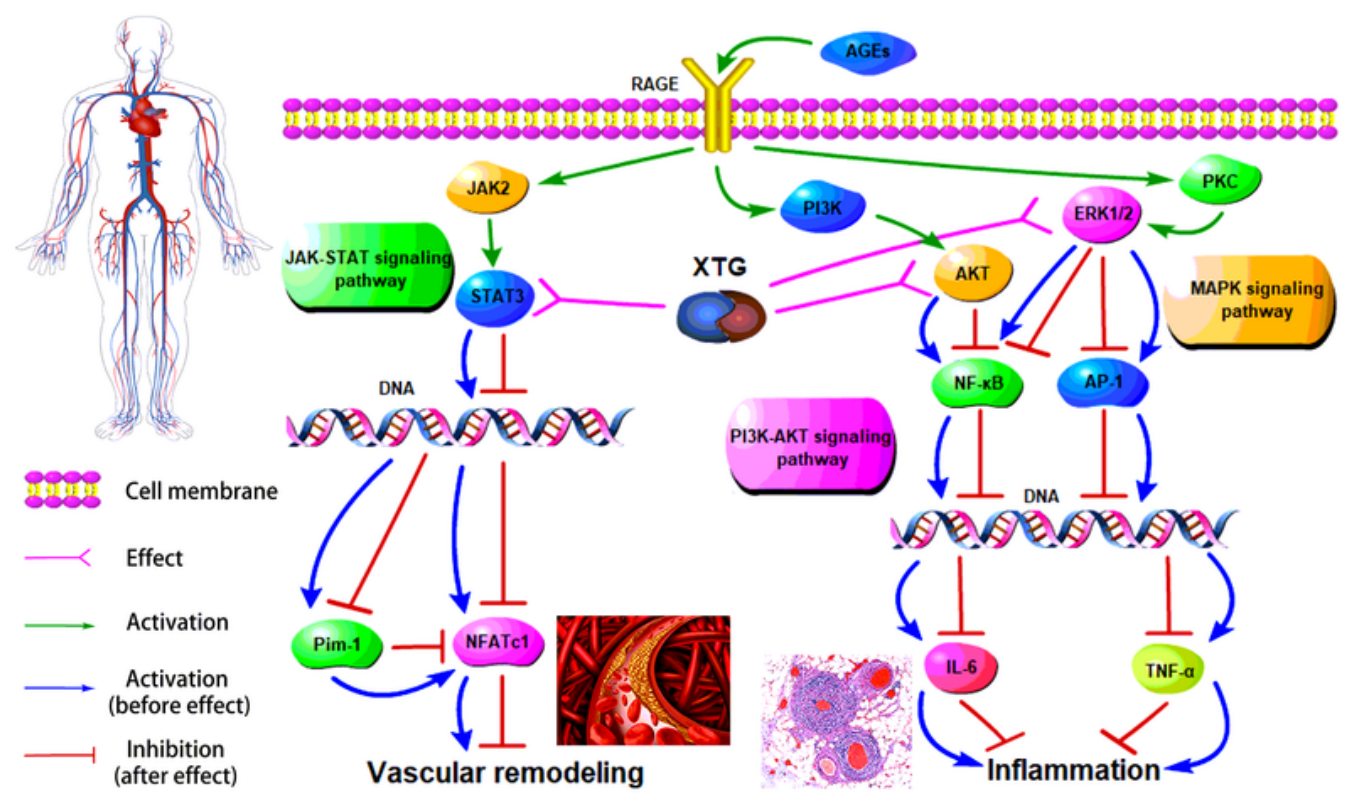

(b)

Figure 6

(a) Modulating AGE-RAGE signaling pathway in diabetic complications of XTG against CHD. Hub targets were colored in red, targets of XTG-CHD were colored in yellow, and other protein targets in the pathway 
were colored in green. (b) Illustration of the crucial biological processes involving putative targets and known therapeutic targets of XTG: Signal transducer and activator of transcription 3 (STAT3), RAC-alpha serine/threonine-protein kinase (AKT1), Mitogen-activated protein kinase 1 (MAPK1), Tumor necrosis factor (TNF), Interleukin-6 (IL6).

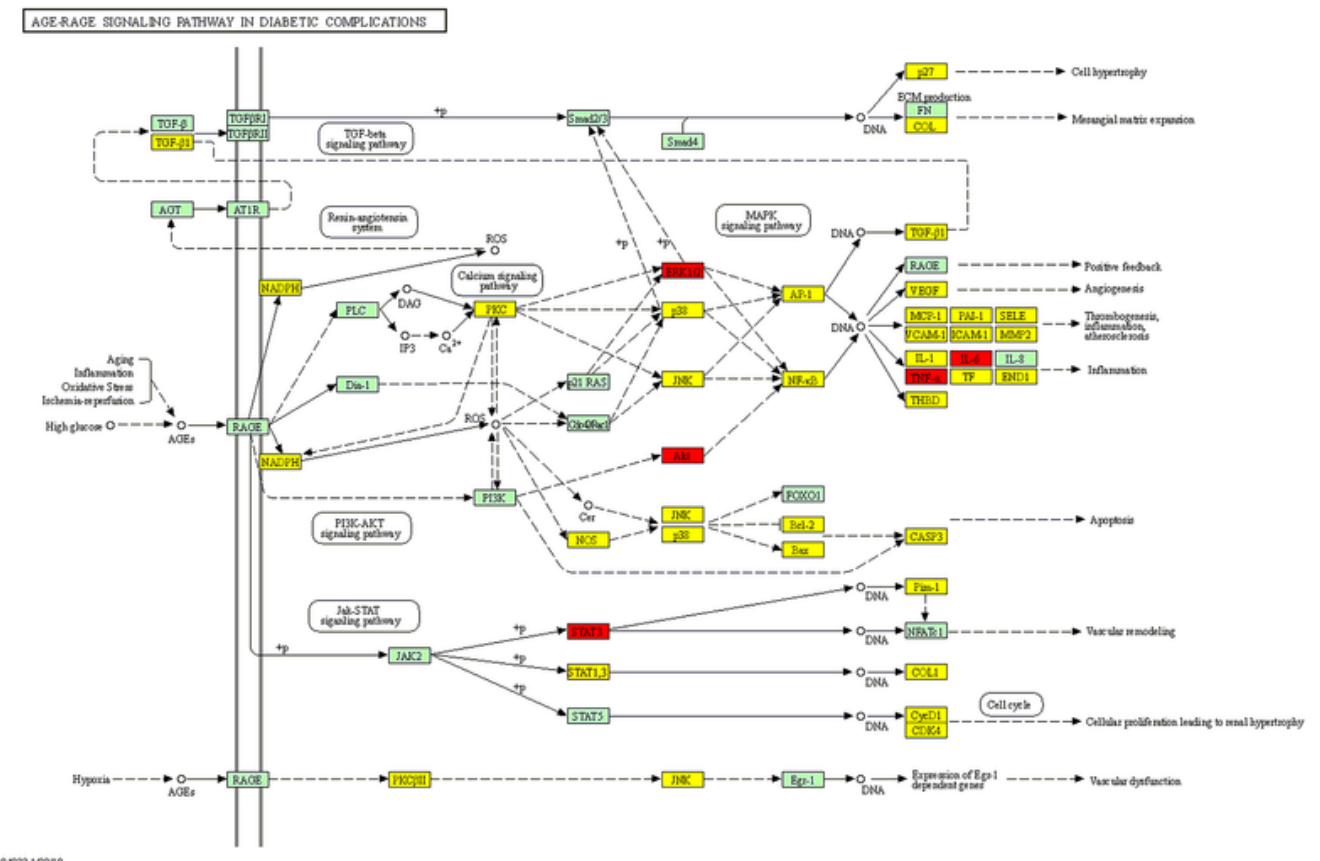

(a)

\section{AGE-RAGE signaling pathway in diabetic complications}
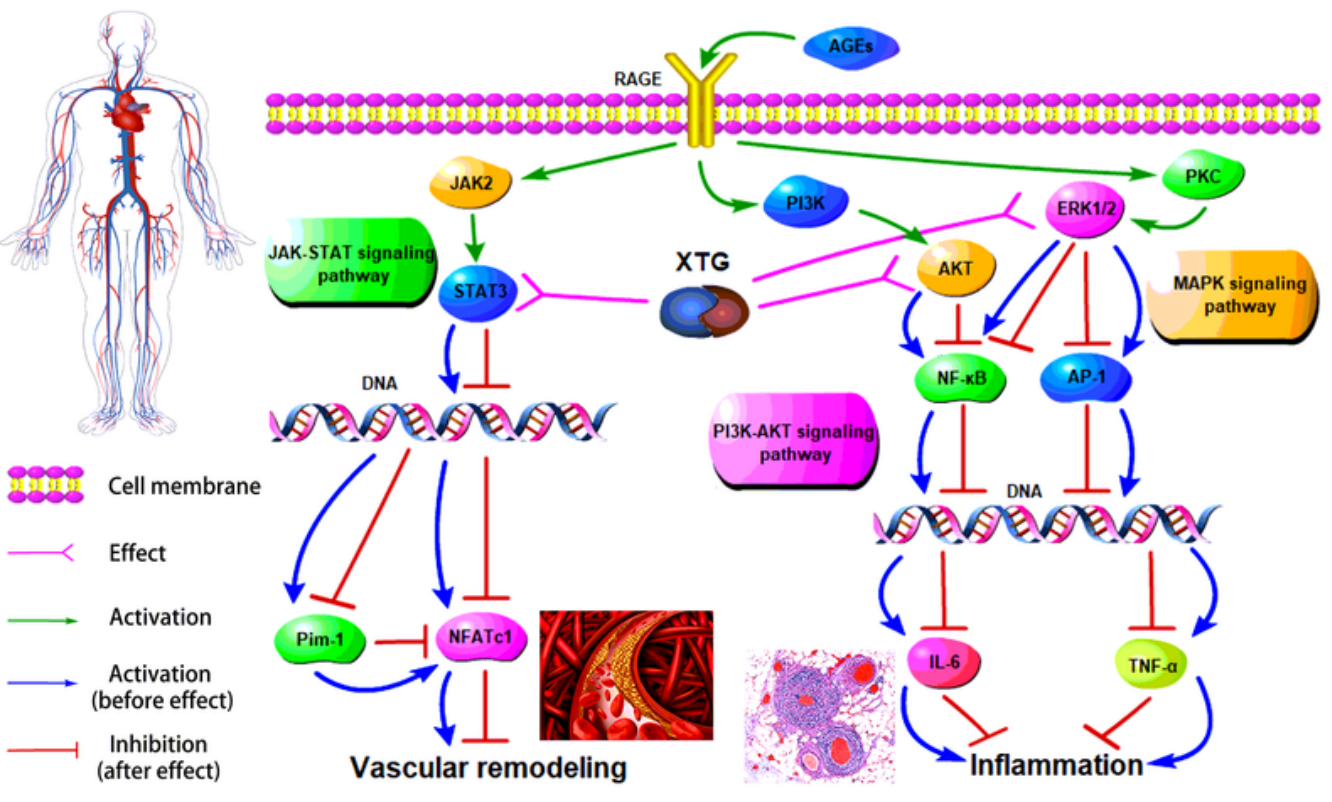

(b)

Figure 6 
(a) Modulating AGE-RAGE signaling pathway in diabetic complications of XTG against CHD. Hub targets were colored in red, targets of XTG-CHD were colored in yellow, and other protein targets in the pathway were colored in green. (b) Illustration of the crucial biological processes involving putative targets and known therapeutic targets of XTG: Signal transducer and activator of transcription 3 (STAT3), RAC-alpha serine/threonine-protein kinase (AKT1), Mitogen-activated protein kinase 1 (MAPK1), Tumor necrosis factor (TNF), Interleukin-6 (IL6).



Fisetin act on IL6 (Affinity $=-7.1 \mathrm{kcal} / \mathrm{mol}$ )

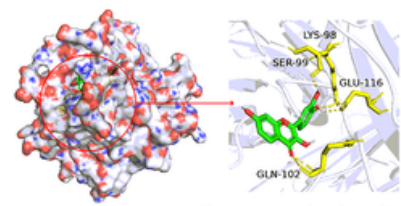

Fisetin act on TNF (Affinity $=-8.8 \mathrm{kcal} / \mathrm{mol}$ )

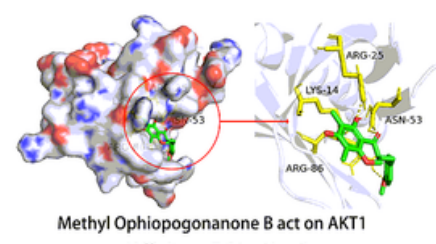

(Affinity $=-7.6 \mathrm{kcal} / \mathrm{mol}$ )

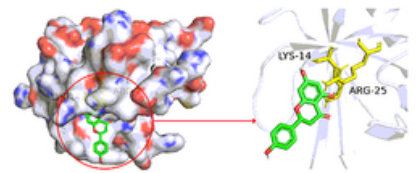

Naringenin act on AKT1 (Affinity $=-6.7 \mathrm{kcal} / \mathrm{mol}$ )

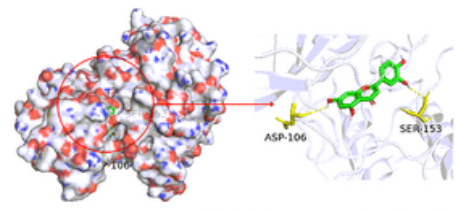

Luteolin act on MAPK1 (Affinity $=-8.6 \mathrm{kcal} / \mathrm{mol}$ )

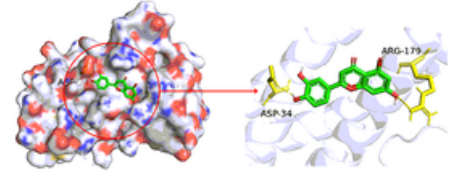

Luteolin act on IL6 (Affinity $=-7.0 \mathrm{kcal} / \mathrm{mol}$ )

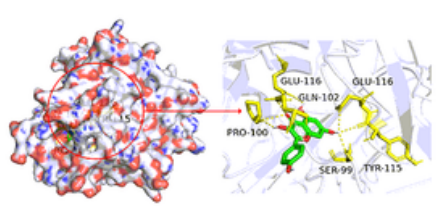

Kaempferol act on TNF (Affinity $=-8.9 \mathrm{kcal} / \mathrm{mol}$ )

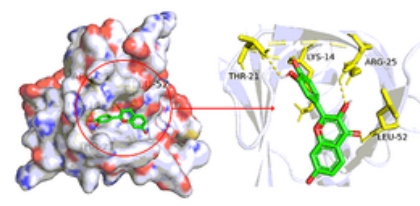

Fisetin act on AKT1 (Affinity $=-6.5 \mathrm{kcal} / \mathrm{mol}$ )

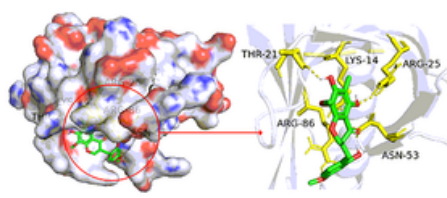

Ophiopogonanone E act on AKT1 (Affinity $=-7.6 \mathrm{kcal} / \mathrm{mol}$ )

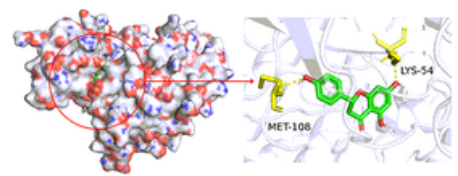

Naringenin act on MAPK1 (Affinity $=-8.7 \mathrm{kcal} / \mathrm{mol}$ )

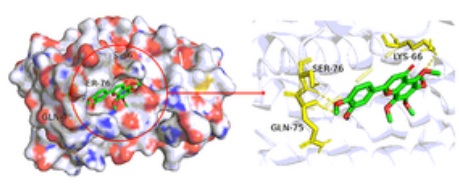

Nobiletin act on IL6 (Affinity $=-5.8 \mathrm{kcal} / \mathrm{mol})$

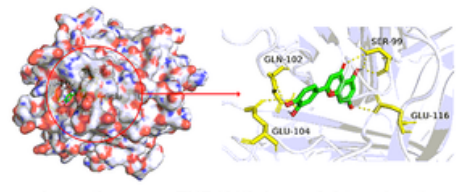

Luteolin act on TNF (Affinity $=-9.0 \mathrm{kcal} / \mathrm{mol}$ )

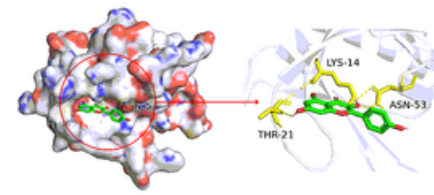

Kaempferol act on AKT1 (Affinity $=-6.5 \mathrm{kcal} / \mathrm{mol}$ )



Quercetin act on AKT1 (Affinity $=-6.5 \mathrm{kcal} / \mathrm{mol}$ )

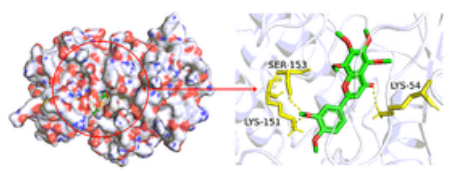

Nobiletin act on MAPK1 (Affinity $=-8.7 \mathrm{kcal} / \mathrm{mol}$ )

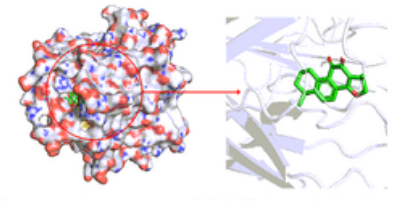

Cryptotanshinone act on TNF (Affinity $=-8.5 \mathrm{kcal} / \mathrm{m}$

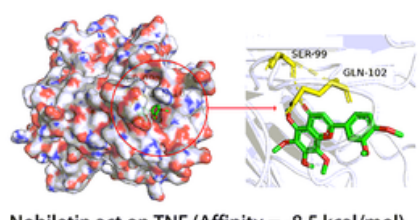

Nobiletin act on TNF (Affinity $=-8.5 \mathrm{kcal} / \mathrm{mol}$ )
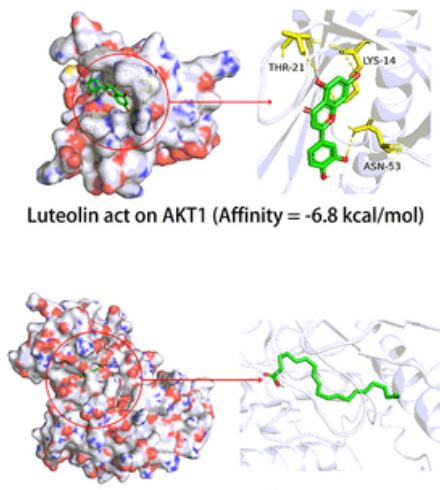

Arachidonic acid act on MAPK1 (Affinity $=-6.8 \mathrm{kcal} / \mathrm{mol}$ )

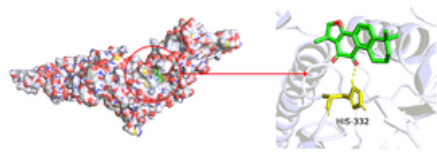

Cryptotanshinone act on STAT3 (Affinity $=-8.0 \mathrm{kcal} / \mathrm{mol}$ )

\section{Figure 7}

Analysis of target-compound docking verification. 


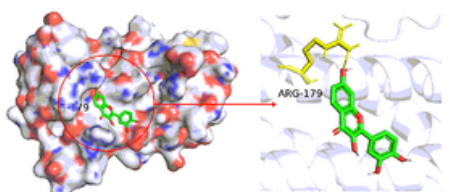

Fisetin act on IL6 (Affinity $=-7.1 \mathrm{kcal} / \mathrm{mol}$ )

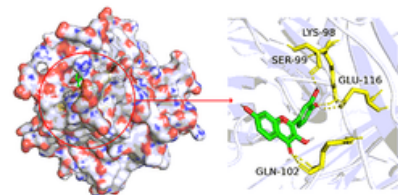

Fisetin act on TNF (Affinity $=-8.8 \mathrm{kcal} / \mathrm{mol}$ )

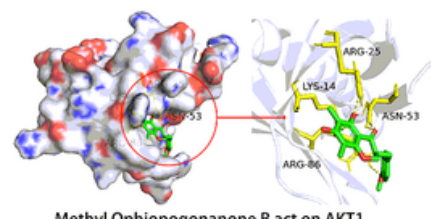

Methyl Ophiopogonanone B act on AKT 1 (Affinity $=-7.6 \mathrm{kcal} / \mathrm{mol}$ )

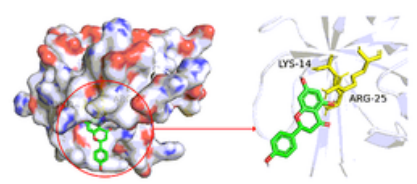

Naringenin act on AKT1 (Affinity $=-6.7 \mathrm{kcal} / \mathrm{mol}$ )

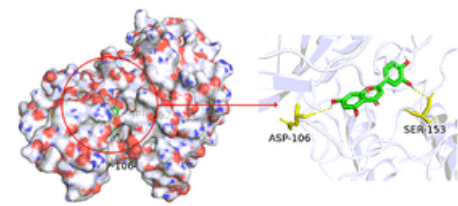

Luteolin act on MAPK1 (Affinity $=-8.6 \mathrm{kcal} / \mathrm{mol}$ )

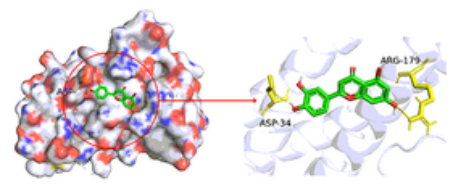

Luteolin act on IL6 (Affinity $=-7.0 \mathrm{kcal} / \mathrm{mol}$ )

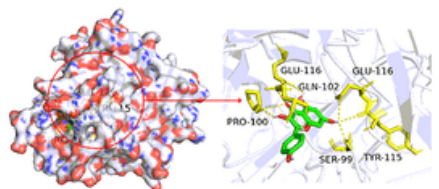

Kaempferol act on TNF (Affinity $=-8.9 \mathrm{kcal} / \mathrm{mol}$ )



Fisetin act on AKT1 (Affinity $=-6.5 \mathrm{kcal} / \mathrm{mol}$ )

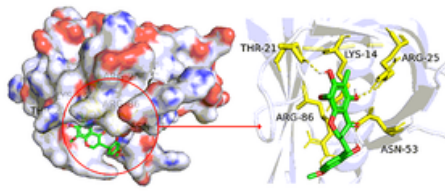

Ophiopogonanone E act on AKT1 (Affinity $=-7.6 \mathrm{kcal} / \mathrm{mol}$ )

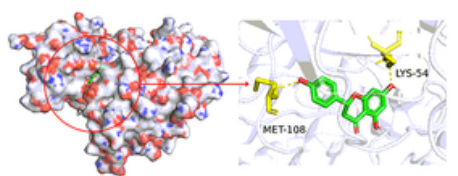

Naringenin act on MAPK1 (Affinity $=-8.7 \mathrm{kcal} / \mathrm{mol}$ )

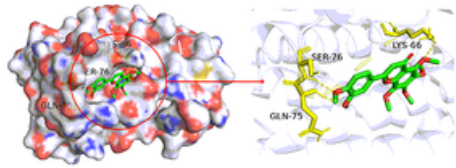

Nobiletin act on IL6 (Affinity $=-5.8 \mathrm{kcal} / \mathrm{mol}$ )

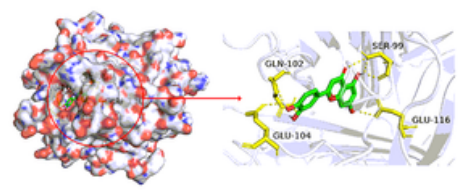

Luteolin act on TNF (Affinity $=-9.0 \mathrm{kcal} / \mathrm{mol}$ )

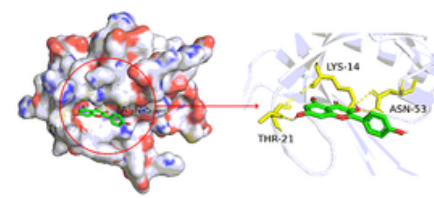

Kaempferol act on AKT1 (Affinity $=-6.5 \mathrm{kcal} / \mathrm{mol}$ )



Quercetin act on AKT1 (Affinity $=-6.5 \mathrm{kcal} / \mathrm{mol}$ )

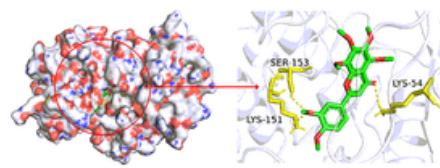

Nobiletin act on MAPK1 (Affinity $=-8.7 \mathrm{kcal} / \mathrm{mol}$ )

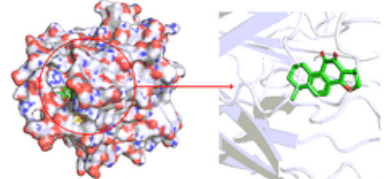

Cryptotanshinone act on TNF (Affinity $=-8.5 \mathrm{kcal} / \mathrm{m}$

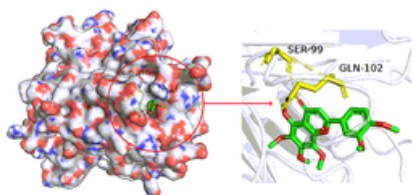

Nobiletin act on TNF (Affinity $=-8.5 \mathrm{kcal} / \mathrm{mol}$ )
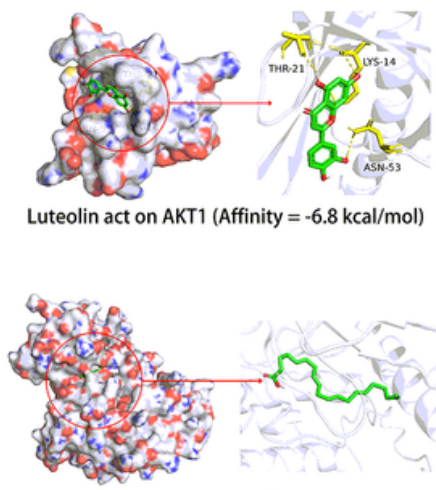

Arachidonic acid act on MAPK1 (Affinity $=-6.8 \mathrm{kcal} / \mathrm{mo}$ )

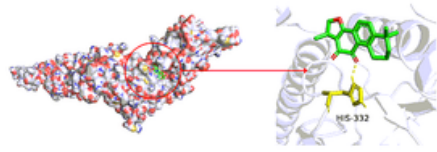

Cryptotanshinone act on STAT3 (Affinity $=-8.0 \mathrm{kcal} / \mathrm{mol}$ )

\section{Figure 7}

Analysis of target-compound docking verification. 


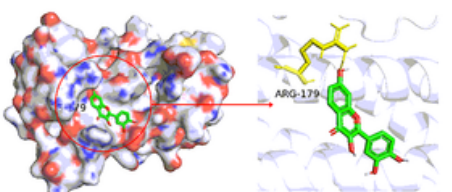

Fisetin act on IL6 (Affinity $=-7.1 \mathrm{kcal} / \mathrm{mol}$ )

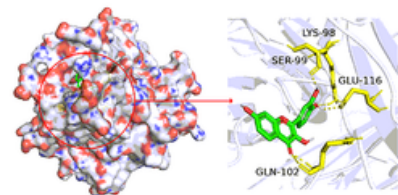

Fisetin act on TNF (Affinity $=-8.8 \mathrm{kcal} / \mathrm{mol}$ )

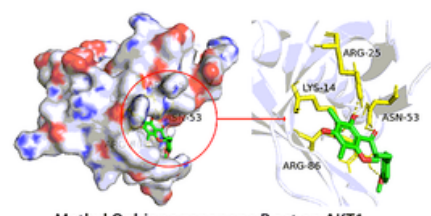

Methyl Ophiopogonanone B act on AKT (Affinity $=-7.6 \mathrm{kcal} / \mathrm{mol}$ )

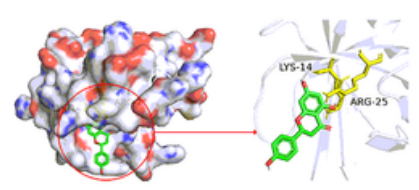

Naringenin act on AKT1 (Affinity $=-6.7 \mathrm{kcal} / \mathrm{mol}$ )

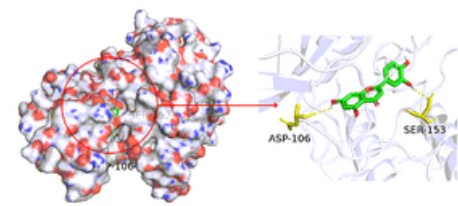

Luteolin act on MAPK1 (Affinity $=-8.6 \mathrm{kcal} / \mathrm{mol}$ )

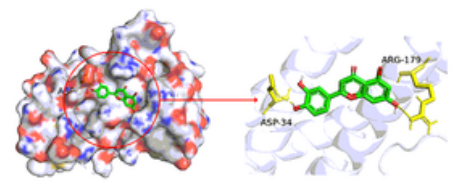

Luteolin act on IL6 (Affinity $=-7.0 \mathrm{kcal} / \mathrm{mol}$ )

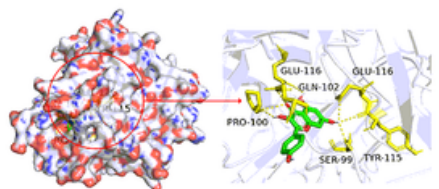

Kaempferol act on TNF (Affinity $=-8.9 \mathrm{kcal} / \mathrm{mol}$ )

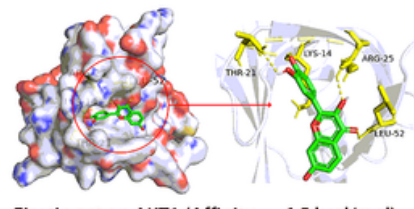

Fisetin act on AKT1 (Affinity $=-6.5 \mathrm{kcal} / \mathrm{mol}$ )

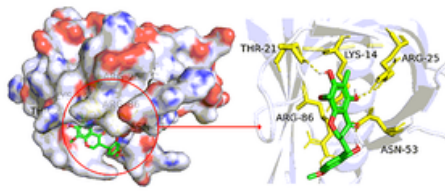

Ophiopogonanone E act on AKT1 (Affinity $=-7.6 \mathrm{kcal} / \mathrm{mol}$ )

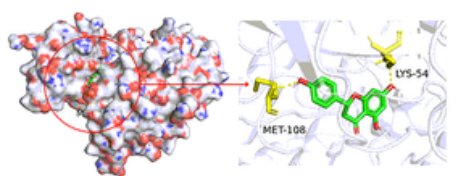

Naringenin act on MAPK1 (Affinity $=-8.7 \mathrm{kcal} / \mathrm{mol}$ )

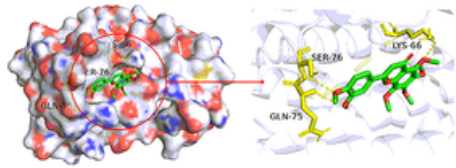

Nobiletin act on IL6 (Affinity $=-5.8 \mathrm{kcal} / \mathrm{mol}$ )

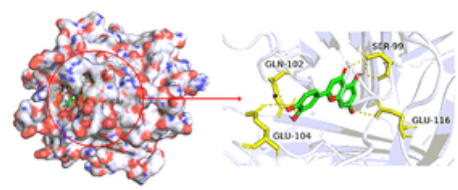

Luteolin act on TNF (Affinity $=-9.0 \mathrm{kcal} / \mathrm{mol}$ )

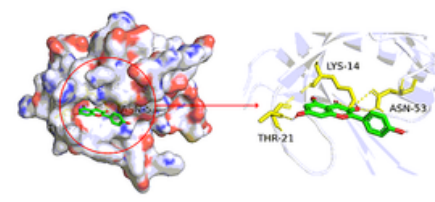

Kaempferol act on AKT1 (Affinity $=-6.5 \mathrm{kcal} / \mathrm{mol}$ )

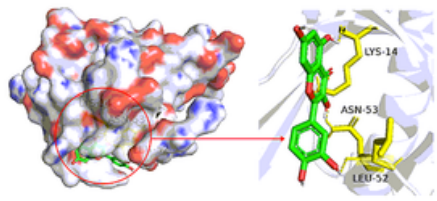

Quercetin act on AKT1 (Affinity $=-6.5 \mathrm{kcal} / \mathrm{mol}$ )

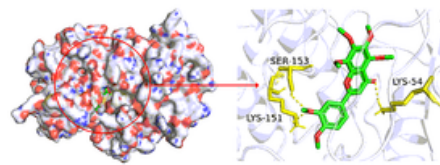

Nobiletin act on MAPK1 (Affinity $=-8.7 \mathrm{kcal} / \mathrm{mol}$ )

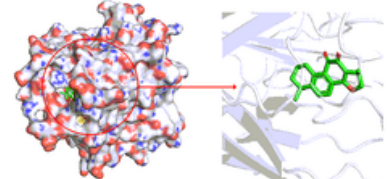

Cryptotanshinone act on TNF (Affinity $=-8.5 \mathrm{kcal} / \mathrm{m}$

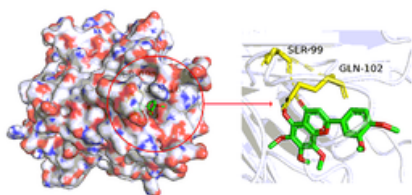

Nobiletin act on TNF (Affinity $=-8.5 \mathrm{kcal} / \mathrm{mol}$ )
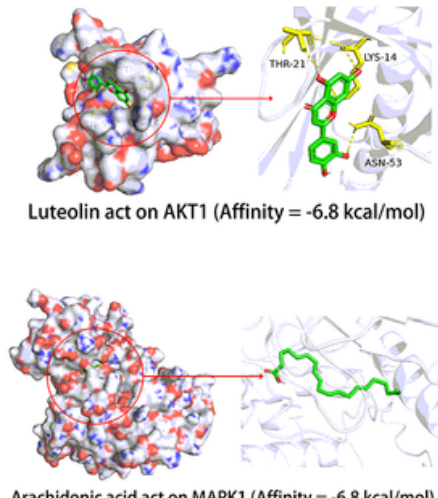

Arachidonic acid act on MAPK1 (Affinity $=-6.8 \mathrm{kcal} / \mathrm{mol}$ )

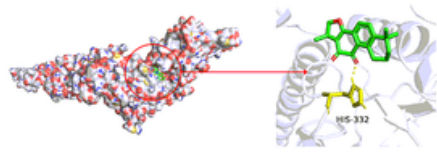

Cryptotanshinone act on STAT3 (Affinity $=-8.0 \mathrm{kcal} / \mathrm{mol}$ )

\section{Figure 7}

Analysis of target-compound docking verification. 


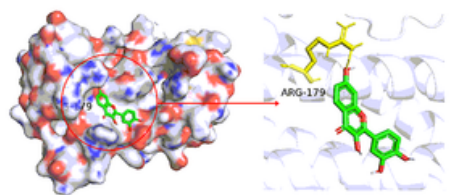

Fisetin act on IL6 (Affinity $=-7.1 \mathrm{kcal} / \mathrm{mol}$ )

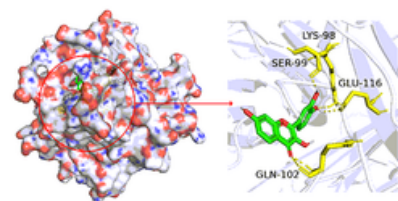

Fisetin act on TNF (Affinity $=-8.8 \mathrm{kcal} / \mathrm{mol}$ )

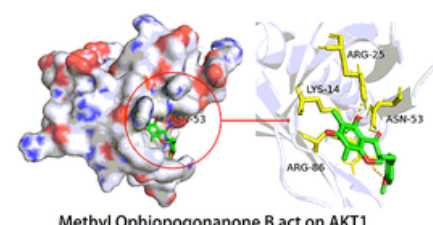

Methyl Ophiopogonanone B act on AKT1 (Affinity $=-7.6 \mathrm{kcal} / \mathrm{mol}$ )

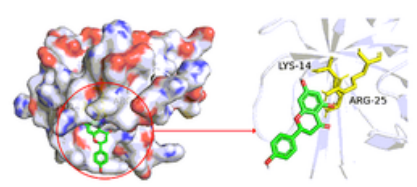

Naringenin act on AKT1 (Affinity $=-6.7 \mathrm{kcal} / \mathrm{mol}$ )

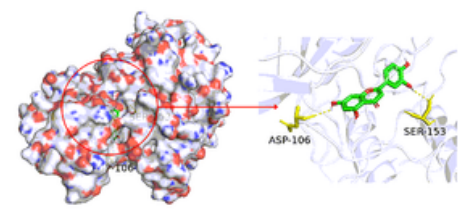

Luteolin act on MAPK1 (Affinity $=-8.6 \mathrm{kcal} / \mathrm{mol}$ )

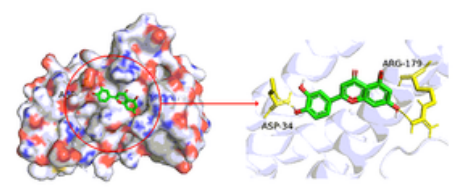

Luteolin act on IL6 (Affinity $=-7.0 \mathrm{kcal} / \mathrm{mol}$ )

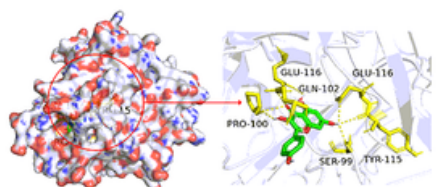

Kaempferol act on TNF (Affinity $=-8.9 \mathrm{kcal} / \mathrm{mol}$ )
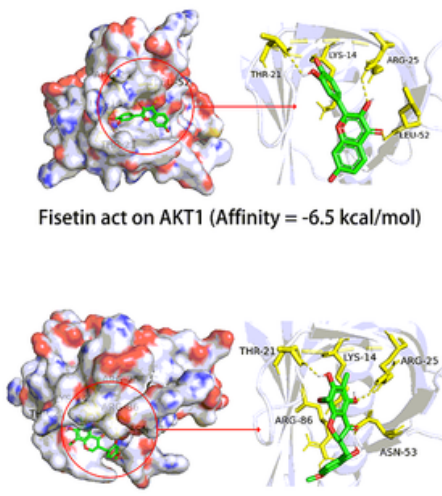

Ophiopogonanone E act on AKT1 (Affinity $=-7.6 \mathrm{kcal} / \mathrm{mol}$ )

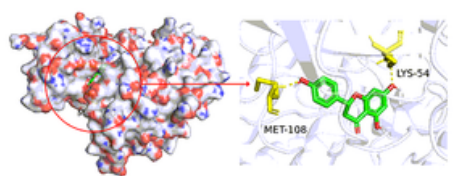

Naringenin act on MAPK1 (Affinity $=-8.7 \mathrm{kcal} / \mathrm{mol}$ )

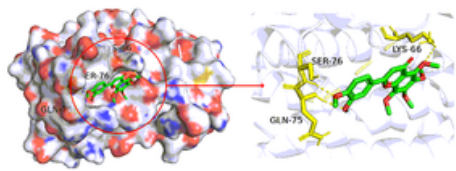

Nobiletin act on IL6 (Affinity $=-5.8 \mathrm{kcal} / \mathrm{mol}$ )

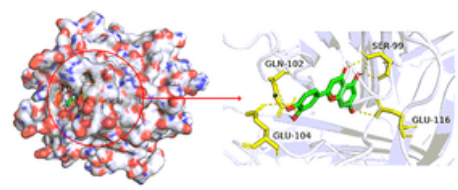

Luteolin act on TNF (Affinity $=-9.0 \mathrm{kcal} / \mathrm{mol}$ )

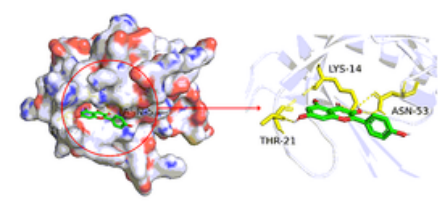

Kaempferol act on AKT1 (Affinity $=-6.5 \mathrm{kcal} / \mathrm{mol}$ )

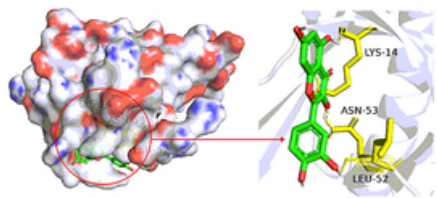

Quercetin act on AKT1 (Affinity $=-6.5 \mathrm{kcal} / \mathrm{mol}$ )

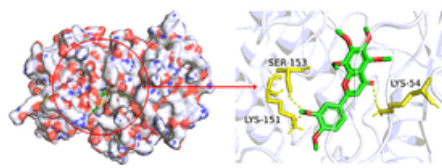

Nobiletin act on MAPK1 (Affinity $=-8.7 \mathrm{kcal} / \mathrm{mol}$ )

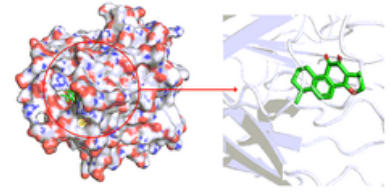

Cryptotanshinone act on TNF (Affinity $=-8.5 \mathrm{kcal} / \mathrm{m}$

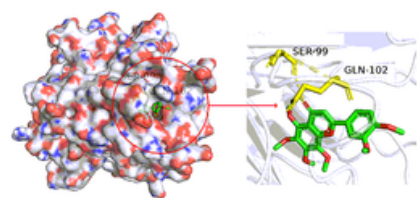

Nobiletin act on TNF (Affinity $=-8.5 \mathrm{kcal} / \mathrm{mol}$ )
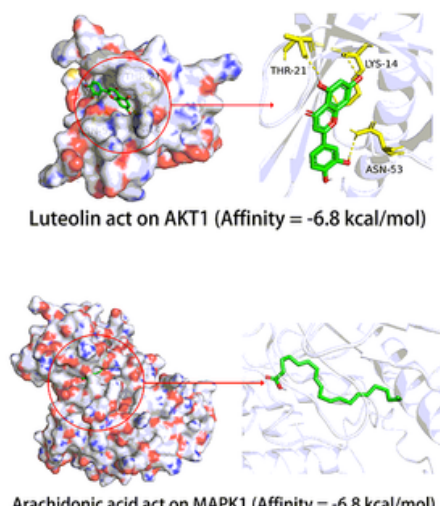

Arachidonic acid act on MAPK1 (Affinity $=-6.8 \mathrm{kcal} / \mathrm{mol}$ )

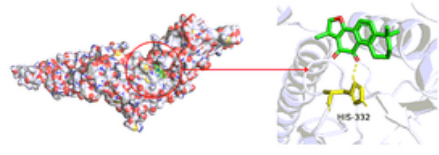

Cryptotanshinone act on STAT3 (Affinity $=-8.0 \mathrm{kcal} / \mathrm{mol}$ )

\section{Figure 7}

Analysis of target-compound docking verification.

\section{Supplementary Files}

This is a list of supplementary files associated with this preprint. Click to download.

- SupplementaryTable1.xlsx

- SupplementaryTable1.xlsx

- SupplementaryTable1.xIsx

- SupplementaryTable1.xIsx

- SupplementaryTable2.xlsx

- SupplementaryTable2.xIsx

- SupplementaryTable2.xIsx 
- SupplementaryTable2.xlsx

- SupplementaryTable3.xlsx

- SupplementaryTable3.xlsx

- SupplementaryTable3.xlsx

- SupplementaryTable3.xlsx

- SupplementaryTable4.xlsx

- SupplementaryTable4.xlsx

- SupplementaryTable4.xlsx

- SupplementaryTable4.xlsx

- SupplementaryTable5.xlsx

- SupplementaryTable5.xIsx

- SupplementaryTable5.xlsx

- SupplementaryTable5.xIsx

- SupplementaryTable6.xlsx

- SupplementaryTable6.xlsx

- SupplementaryTable6.xIsx

- SupplementaryTable6.xlsx

- SupplementaryTable7.xIsx

- SupplementaryTable7.xlsx

- SupplementaryTable7.xlsx

- SupplementaryTable7.xIsx

- SupplementaryTable8.xlsx

- SupplementaryTable8.xlsx

- SupplementaryTable8.xlsx

- SupplementaryTable8.xlsx 